\title{
Hedging (Co)Variance Risk with Variance Swaps*
}

\author{
José Da Fonseca $^{\dagger} \quad$ Martino Grasselli ${ }^{\ddagger} \quad$ Florian Ielpo ${ }^{\S}$
}

First draft: March 4, 2008

This draft: August 25, 2008

\begin{abstract}
In this paper we introduce a new criterion in order to measure the variance and covariance risks in financial markets. In an asset allocation framework with stochastic (co)variances, we consider the possibility to invest also in variance swaps, that are assets which span the volatility as well as the co-volatility risks. We provide explicit solutions for the portfolio optimization problem. We then compare the optimal wealths obtained with and without (co)volatility products leading to the same expected utility. We use the spread between these wealths as a criterion in order to measure the impact of (co)variance risk. Using real data on major indexes and this criterion, we find that the impact of (co)variance risk is huge in the market. This is consistent with the fast development of variance and covariance-based markets.
\end{abstract}

Keywords: Wishart Affine Stochastic Correlation model, complete and incomplete markets, variance swaps, optimal portfolio choice.

JEL codes: G11, G12.

\section{Introduction}

This paper deals with the problem of quantifying and hedging the risks steaming from the stochasticity of assets variances as well as from their correlations. The very fast

\footnotetext{
*The authors are thankful to the participants of the CREST seminar (Paris, 2008), of the Bachelier Finance Society (Imperial College, London, 2008), to the local seminar of Natixis (Paris, 2008), of the Second Annual Risk Management Conference (Singapour, 2008) and of the Computing in Economic and Finance Conference (Paris, 2008) for their remarks. We are particularly thankful to Nicole El Karoui, Christian Gouriéroux, Fulvio Pegoraro and Iuniu Stef. All remaining errors are ours.

†Ecole Supérieure d'Ingénieurs Léonard de Vinci, Département Mathématiques et Ingénierie Financière, 92916 Paris La Défense, France. Email: jose.da_fonseca@devinci.fr and Zeliade Systems, 56, Rue Jean-Jacques Rousseau 75001 Paris

${ }^{\ddagger}$ Università degli Studi di Padova, Dipartimento di Matematica Pura ed Applicata, Via Trieste 63, Padova, Italy.E-mail: grassell@math.unipd.it and ESILV.

${ }^{\S}$ Centre d'Economie de la Sorbonne, 106 Boulevard de l'Hopital, 75013 Paris, France. E-mail: ielpo@ces.ens-cachan.fr and Dexia S.A., Passerelle des Reflets, La Défense, France.
} 
development of the variance swap market together with other OTC correlation products (like Correlation Swaps) clearly indicates that variance and covariance risk is indeed important to portfolio managers, thus leading to a huge demand of hedging such risks. In the portfolio optimization literature, however, the specific hedging demand ${ }^{1}$ for the volatility-covolatility risks is usually small. The following table summarizes the variance hedging demand (in terms of $\%$ on the Merton myopic portfolio) found in the empirical literature on the major indexes ${ }^{2}$ :

\begin{tabular}{ccccccc}
\hline \hline & Liu05 & CV05 & LEW07 & BPT06 (declared) & BPT06 (correct) & DGI08 \\
\hline \hline SP500 & Very small & 2.13 & 2.50 & 10 & 0.77 & 0.63 \\
DAX & - & - & - & 11 & 1.01 & 0.89 \\
\hline \hline
\end{tabular}

For most of the contributions, the hedging demand for stochastic volatility is small ${ }^{3}$. Even in the Buraschi et al. (2006) paper, the advocated ratio is around to $10 \%$, which is however small: the very fast development of the variance swap market clearly indicates that variance and covariance risk is indeed important to portfolio managers. In this paper we introduce a new criterion to measure the (co)variance risk. We show that hedging demand-based criteria are misleading when dealing with variance and covariance risks in financial markets. We measure the (co)variance risk by comparing the spread between the initial wealths required to obtain the same final utility in an incomplete and completed market case. According to Leippold et al. (2007), we show that when completing the market by introducing volatility products (namely variance swaps), the additional initial wealth can be dramatically large. The additional expected utility based criterion provides a better measure of covariance risks than the size of hedging demand: the claim for (co)volatility hedging exceeds by far what has been found in the previous literature. The use of variance swap makes it possible to

\footnotetext{
${ }^{1}$ In the Merton optimal allocation framework with constant investment opportunity set, the optimal allocation rule is known as the myopic portfolio. When the investment opportunity set is stochastic due to the presence of stochastic volatility, an additional term is added to the myopic portfolio and is known as the (co)volatility hedging demand. This additional term is the ratio of the additional term linked to the stochasticity of the covariances to the original myopic portfolio. It thus represents the modification percentage of the optimal portfolio when covariance are stochastic.

${ }^{2}$ The first column is deduced by the paper of Liu (2005) and Liu (2007); the second column from Chacko and Viceira (2005), the third column contains the results presented in Leippold et al. (2007) (the results presented are the one obtained for the 1996-2007 sample); the fourth column is taken from Buraschi et al. (2006); the fifth column contains the results of Buraschi et al. (2006) when we recompute their solution using the estimates and dataset presented in their paper, along with their model; the sixth column contains the values corresponding to the WASC model adopted in our paper. We detail in the empirical Section the computations of the table.

${ }^{3}$ The only exception is the recent paper of Buraschi et al. (2006). However, when recomputing their solutions using their estimates, we obtain a totally different - but consistent with past literature - result. There seems to be an empirical mistake in their paper in implementing their solution. A Matlab code providing their solution is available on first author's webpage (and is available upon request). In the appendix, we provide the numerical values taken from Buraschi et al. (2006) used to recompute their solution and the corrected solution.
} 
discuss the (co)variance risk, since this asset is specifically devoted to (co)volatility risk hedging. What is more, this criterion has a way better financial interpretation than the usual hedging demand: we discuss this point extensively in the paper.

We consider an inter-temporal portfolio problem within the Wishart Affine Stochastic Correlation (WASC hereafter) model introduced by Da Fonseca et al. (2007b): in our multi-dimensional model, asset's volatilities evolve stochastically as well as their covolatilities, following a Wishart process recently introduced in finance by Gourieroux and Sufana (2004) and extended by Buraschi et al. (2006). This affine model can be regarded as a multivariate version of the Heston (1993) one: in fact, the volatility matrix is assumed to evolve according to the Wishart dynamics (mathematically developed by Bru (1991)), which represents the matrix analogue of the mean reverting square root process. In addition to the Heston model, it allows for a stochastic conditional correlation, which makes it a very promising process for financial applications.

When dealing with a stochastic (co-)volatility model, it is well known that an intertemporal hedging demand arises in the Merton-like optimal strategy, in order to take into account the fluctuations of the relevant stochastic factors. In the stochastic volatility literature, this phenomenon has been investigated from both theoretical and empirical point of view. Chacko and Viceira (2005) are amongst of the first to study empirically the optimal portfolio problem in a Heston stochastic volatility (incomplete) market setting. In an infinite horizon economy, they considered a recursive utility criterion. They found that the intertemporal hedging demand for reasonable values of the risk aversion parameter is small (about $2 \%$ of the Merton myopic portfolio). Liu (2005) and Liu (2007) considers constant relative risk aversion (CRRA) preferences and he finds similar results.

In a multi-asset setting, Buraschi et al. (2006) extended the analysis of Liu (2005) and Liu (2007) and Chacko and Viceira (2005) to the case where both volatilities and correlation are stochastic. They enhanced the model of Gourieroux and Sufana (2004) by introducing a special correlation structure among asset returns and their (co)volatilities. They obtained a model that is nested within the Wishart Affine Stochastic Correlation model independently introduced in Da Fonseca et al. (2007b) in a option pricing framework. The main message of Buraschi et al. (2006) is that the intertemporal hedging demand coming from the presence of a stochastic correlation is a "non-negligible fraction of the myopic portfolio, which often dominates the pure volatility hedging demand" (around $15 \%$ ). They conclude with the fact that correlation risk is high when compared to volatility risk.

However, it is well known that variance risk and hedging demand are different concepts. This is intuitive. For example, in the Heston model framework, if the volatility 
is independent of the asset returns, the hedging demand is known to be equal to zero. Nevertheless, in such a framework, there is obviously a variance risk that should be hedged some way. The zero-hedging demand only reflects the fact that variance risk cannot be hedged solely by trading the underlying (see e.g. Hull (2008)). The usual way to hedge such risk is to introduce assets that are known to depend on the additional risk factors. In a stochastic volatility framework, the natural choice would consist in Variance Swaps, since they are (linear) forward contracts on the realized variance, thus they can span the uncertainty coming from the stochastic volatility. Leippold et al. (2007) follow this approach in the Heston (1993) framework. They quantify the variance risk by considering the additional utility coming from the introduction of Variance Swaps in the market. They compare the optimal allocations strategies in both complete and incomplete market and find that they can be drastically different in the two cases. In particular, in the complete market the optimal demand in the risky asset turns out to be a constant proportion of the wealth as in the usual Merton-like solution. What is more, the additional utility coming from the introduction of variance swaps can be very large even when intertemporal hedging demand is small, thus confirming that the latter cannot be considered as a measure for quantifying the variance risk.

In the first part of our paper we extend the idea of Leippold et al. (2007) to the case where variances and correlations are stochastic. We consider a market in which an agent endowed with a CRRA utility over terminal wealth holds a portfolio made of risky assets and variance swaps. The latter assets span the uncertainty coming from the stochastic variance-covariance matrix of the assets returns. Notice that in our framework Variance Swaps are also correlation products since they involve all the elements of the variance-covariance matrix. In the complete and incomplete market cases we find the optimal demand in risky assets and in the variance swaps. We then propose to test empirically the solutions, using a real dataset on S\&P500 and DAX. Using both the underlying and variance swaps, we estimate the WASC model under both the historical and pricing measure. The empirical results unfold as follows: first, in the incomplete market case, we recover results similar to those found in the previously mentioned literature. Second, in the complete market case, we find a huge change in both optimal allocation strategy and associated expected utility. Roughly speaking, our results indicate that without variance swaps, the agent needs about 250 times his initial wealth in order to obtain the same expected utility level as in the complete market case, thus indicating a significant (co)variance risk in the market.

The paper is organized as follow. In Section 2, we present the stochastic correlation model used as a framework for this paper. In Section 3, we present the optimal portfolio allocation problem in both incomplete and complete market case. In Section 4 we review the estimation methodology along with the empirical results obtained in the paper. Section 5 concludes, while we gathered the technical proofs in the Appendix. 


\section{The model}

The model we adopt for describing the economy is the Wishart Affine Stochastic Correlation (WASC hereafter) model introduced in Da Fonseca et al. (2007b). In this multiasset market returns's volatilities evolve stochastically as well as their co-volatilities. In fact, quadratic covariations evolve as additional stochastic factors. Moreover, according to Da Fonseca et al. (2007b), in order to be consistent with the APT Factor Model, the dynamics of all variance-covariance factors are supposed to be a linear-affine. In this way we obtain the genuine multi-dimensional version of the the celebrated Heston (1993) model, where the square root variance process is replaced by a (matrix valued) Wishart process investigated by Bru (1991) and introduced in finance by Gourieroux and Sufana (2004) in a multi-asset framework. Let us briefly recall the main assumptions underlying the WASC model:

Assumption 2.1. The continuous time diffusive Factor Model is considered to be a linear-affine stochastic factor model with respect to assets' returns $Y_{i t}^{*}$ and variancecovariance factors $\Sigma_{k l}$.

In line with Da Fonseca et al. (2007b), in this model (observed) returns and their variances can be explained as linear combinations of fundamental risk factors (including covariance risks) whose evolution is still a linear-affine process.

As described in Gourieroux and Sufana (2004), it is possible to describe the stochastic evolution of a generic stochastic positive definite variance-covariance matrix within a linear-affine model with a non linear (cone) state space and Grasselli and Tebaldi (2008) state sufficient parametric conditions in order to obtain an admissible process preserving the cone state space formed by the set of positive definite symmetric matrices. For this reason we retain the Gourieroux and Sufana (2004) specification of the variance process:

Assumption 2.2. The stochastic covariance matrix follows a Wishart process.

The instantaneous variance covariance of the risky assets is a matrix $\Sigma_{t}$ which is assumed to satisfy the following dynamics:

$$
d \Sigma_{t}=\left(\Omega \Omega^{\top}+M \Sigma_{t}+\Sigma_{t} M^{\top}\right) d t+\sqrt{\Sigma_{t}} d W_{t} Q+Q^{\top}\left(d W_{t}\right)^{\top} \sqrt{\Sigma_{t}},
$$

where $\Omega \Omega^{\top}=\beta Q^{\top} Q, \quad \beta>n-1$ and $\Omega$ is invertible.

Equation (1) characterizes the Wishart process introduced by Bru (1991), see also Da Fonseca et al. (2007a) for some financial interpretation of the parameters. Note that the first condition requires a proportionality relation between $\Omega \Omega^{\top}$ and $Q^{\top} Q$ and provides a strong reduction on the number of free parameters in the model. The proportionality (Gindikin) parameter $\beta$ is constrained to be larger than $n-1$. When $n=1$ we recover the standard condition granting the positivity of the process for the square-root diffusion model. In full analogy with the square-root diffusion process, the 
term $\Omega \Omega^{\top}$ is related to the expected long-term variance-covariance matrix $\Sigma_{\infty}$ through the solution to the following linear (Liapunov) equation:

$$
-\Omega \Omega^{\top}=M \Sigma_{\infty}+\Sigma_{\infty} M^{\top}
$$

In a one dimensional model the matrix $M$ would collapse to the mean reversion velocity; in the multivariate situation the matrix $M$ is still responsible for the mean reversion effects, however in case of a non diagonal $M$ it will also generate an effective dynamic interaction between different asset volatilities. Similarly the matrix $Q$ is the multivariate counterpart of the volatility of volatility parameter in the Heston model.

Finally we consider the following extension of all previously known (affine) models:

Assumption 2.3. Brownian motions of the assets' returns and those driving their instantaneous covariance matrix are linearly correlated.

This extension is motivated by the well known fact that within the Heston (1993) model it is possible to approximately reproduce observed skews only introducing a non zero correlation between the innovation of returns and the innovation driving variance. In the same way it is reasonable to expect that these correlations are needed in order to fully capture effects of dynamic stochastic correlations on multiple asset options.

In formulas, we consider a $n$-dimensional risky asset $S_{t}$ whose dynamics under the historical probability measure $\mathbb{P}$ are given by

$$
d S_{t}=\operatorname{diag}\left[S_{t}\right]\left[\mu_{t}(\omega) d t+\sqrt{\Sigma_{t}} d Z_{t}\right],
$$

where $Z_{t} \in \mathbb{R}^{n}$ is a vector Brownian motion.

Under the above assumptions, Da Fonseca et al. (2007b) showed that the correlation matrix among the Brownian motions has a very special and parsimonious form:

Proposition 2.1. (Da Fonseca et al. (2007b)) Assumption 2.3 and Assumption 2.2 imply that

$$
d Z_{t}=\sqrt{1-\rho^{\top} \rho} d B_{t}+d W_{t} \rho .
$$

where $\rho \in[-1,1]^{n}$ and $\rho^{\top} \rho \leq 1$.

Remark 2.1. The market model introduced by Gourieroux and Sufana (2004) corresponds to the case $\rho=0$, for which the Brownian motions of assets' returns are assumed to be independent of the ones driving the volatility matrix, thus implying a flat skew effect on the implied volatility structure of vanilla options as discussed in Da Fonseca et al. (2007b). The model of Buraschi et al. (2006) corresponds to the case $\rho=(1,0)^{\top}$, that is the Brownian motion driving the asstes' returns is generated by the (matrix) Brownian motion of the volatility process, so that their model can be viewed as the multi-dimensional version of the Heston-Nandi model, in which there is a perfect correlation between these noises. 


\subsection{The risk premia}

The WASC model naturally accommodates a flexible parametrization of positive stochastic risk premia depending also on covariation levels. Following Gourieroux and Sufana (2004), in line of principle on should consider the general form for the risk premium of the risky assets, which is assumed to be a linear-affine combination of the risk factors. From the Riesz representation theorem of linear functionals, this implies the following general specification:

$$
\mu_{t}^{k}=r+\operatorname{Tr}\left[\Theta_{t}^{k} \Sigma_{t}\right], \quad k=1, \ldots, n,
$$

for some deterministic matrices $\Theta_{t}^{k} \in M_{n}$. However, in order to grant the linear-affinity of the model (which implies the analytical tractability) under both the historical and risk neutral probability measures, some additional constraints on the functional form of the risk premia should be introduced. Technically speaking, the Girsanov change of drift for the Brownian motion $Z$ inherits some restrictions on the transforms for both $B$ and $W$. Such constraints are in general quite difficult to manage and we will do not investigate here the (delicate) question about the most general expression for the risk premium preserving the linear-affinity of the model: this is why instead of assuming the general form as in (3), we adopt the so called completely affine parametrization (in the terminology of Duffee (2002)) for the market price of risk as in Da Fonseca et al. (2007b), thus leading to a price deflator whose dynamic is given by the following SDE:

$$
\frac{d H_{t}}{H_{t}}=-r d t+\operatorname{Tr}\left[\Lambda^{\top} \sqrt{\Sigma_{t}} d W_{t}\right]-l^{\top} \sqrt{\Sigma_{t}} d B_{t},
$$

where $\Lambda \in M_{n \times n}(\mathbb{R}), l \in \mathbb{R}^{n}$. In other words, the Girsanov changes of drifts for the Brownian motions $W, B$ are parametrized by a matrix $\Lambda$ and a vector $l \in \mathbb{R}^{n}$ such that

$$
\begin{aligned}
d W_{t}^{\mathbb{Q}} & =d W_{t}-\sqrt{\Sigma_{t}} \Lambda d t, \\
d B_{t}^{\mathbb{Q}} & =d B_{t}+\sqrt{\Sigma_{t}} l d t,
\end{aligned}
$$

where $W_{t}^{\mathbb{Q}} \in M_{n}$ (resp. $B_{t}^{\mathbb{Q}}$ ) is a matrix (resp. vector) Brownian motion under the risk neutral measure $\mathbb{Q}$. This means that the SDE describing the WASC model under the risk neutral measure $\mathbb{Q}$ is related to the original $\mathbb{P}$-process by the following reparametrization:

$$
M^{\mathbb{Q}}=M+Q^{\top} \Lambda^{\top},
$$

so that the risk premium associated to the risky assets is given by (see also Da Fonseca et al. (2007b))

$$
\Sigma_{t} \lambda_{0}=\Sigma_{t}\left(\sqrt{1-\rho^{\top} \rho} l-\Lambda \rho\right)
$$

\subsection{Variance and Covariance Swaps}

In a Heston-like framework, Leippold et al. (2007) introduced the possibility to invest wealth into a Variance Swap, that is a linear contract on the realized variance which is 
perfectly correlated with the Brownian motion driving the variance process. Leippold et al. (2007) showed that the optimal investment strategy with and without a variance swap changes dramatically, even if the intertemporal hedging component in the optimal demand in the risky assets remains small in absolute value (according to Chacko and Viceira 2005 and Liu 2005). In fact, the size of the hedging demand indicates what can be done by just investing into the risky asset, but it tells very little about the size of the variance risk. For example, if the volatility process is independent of the asset's noise, it is well known that the hedging demand is equal to zero (then the optimal strategy turns out to be the classic Merton's one, see e.g. Example 7.4 p.305 in Karatzas and Shreve (1998) for the very general result), but of course the variance risk could be high and it should be computed in another way. In order to quantify the Variance Risk, we suggest to compute the additional utility coming from the introduction of the variance swaps. Another strategy, followed by Leippold et al. (2007), consists in changing the RRA parameter in order to obtain similar hedge ratios when completing the market with the variance swap.

Following the idea of Leippold et al. (2007), we introduce some non redundant linear contracts which span the uncertainty coming from the stochastic variance-covariance matrix.

The first class of linear contracts consists in Variance Swaps, which are forward contracts on the realized variance. As commonly used in practice, the variance swaps price stands for the strike price of the corresponding forward contract on the realized variance. The variance swap rate on the $i-t h$ asset with maturity $T$ at time $t$ is given by:

$$
V S^{i i}(t, T)=\frac{1}{T-t} E_{t}^{\mathbb{Q}}\left[\int_{t}^{T} \Sigma_{u}^{i i} d u\right]
$$

where $\Sigma_{u}^{i i}$ denotes the diagonal element of the variance-covariance matrix $\Sigma$ and is associated to the (instantaneous) variance of the $i-$ th asset.

Since in the WASC model not only variances but also correlations are stochastic, we will consider a second class of derivatives, namely Covariance Swaps, in order to span the uncertainty coming from the stochastic correlations. In analogy with the variance swap, the Covariance Swap rate between assets $S_{i}$ and $S_{j}$ is given by

$$
V S^{i j}(t, T)=\frac{1}{T-t} E_{t}^{\mathbb{Q}}\left[\int_{t}^{T} \Sigma_{u}^{i j} d u\right] .
$$

Contrarily to Variance Swaps, Covariance Swap rates are not quoted in the market. However, Carr and Corso (2001) show how to construct a synthetic Covariance Swap by using the volatility products quoted in the market, such that pricing and hedging 
of (synthetic) Covariance Swaps can be performed efficiently. Since we work under the historical measure, we also need to estimate the risk premia of Covariance Swaps: in this case we will perform a Kalman filter estimation on the available variance swap rates in order to estimate also the covariance swaps' risk premia, in line with Leippold et al. (2007).

Remark 2.2. Estimating Covariance Swaps' risk premia using Variance Swaps prices could be quite surprising at a first attempt, but it is important to notice that in the WASC model Variance Swaps are correlation products since their prices involve all the information of the volatility-covolatility matrix (that is also the off-diagonal elements of the matrix $\Sigma$ ). In this sense it is quite natural to estimate correlation products by using (co-)volatility products like Variance Swaps, and this also explains the title of our paper.

We denote with $V S(t, T)$ the matrix (co-)variance swap rate whose elements are $V S^{i j}(t, T)$. Since variance and covariance swaps are linear functions of the variance matrix $\Sigma_{t}$, we can easily deduce the (co-)variance swap rate by solving a linear ODE (which is much simpler than the procedure based on the characteristic function proposed by Leippold et al. (2007)). As shown in the Appendix, the (co-)variance swap rates are given by:

$$
V S^{i j}(t, T)=\frac{1}{T-t} \operatorname{Tr}\left[\Sigma_{t} \int_{t}^{T} L_{u}^{i j} d u\right]+\frac{1}{T-t} \int_{t}^{T} \operatorname{Tr}\left[e_{i j} e^{(u-t) M^{\mathbb{Q}}} A_{21} d u\right],
$$

where the deterministic matrix $L$ is given by

$$
L_{u}^{i j}=e^{(u-t) M^{\mathbb{Q}^{\top}}} e_{i j} e^{(u-t) M^{\mathbb{Q}}}
$$

( $e_{i j}$ denotes the $i j$-element of the canonical basis of the set of square matrices) while the deterministic matrix $A_{21}$ is provided in the Appendix.

In conclusion, the variance and covariance swap rates (as well as their dynamics) are available in closed form in the Wishart specification for the variance-covariance matrix.

\section{The optimal allocation problem}

\subsection{The portfolio problem in the incomplete market}

In the first part of our analysis we consider the case where the (co-)variance swaps are not available in the market, so that the agent invests his initial wealth $X_{0}$ into the (incomplete) financial market consisting only in the risky assets $S_{t}^{1}, \ldots, S_{t}^{n}$. The dynamics of the (self financing) wealth process is given by

$$
d X_{t}=X_{t}\left(r+\pi_{t}^{\top} \Sigma_{t} \lambda_{0}\right) d t+X_{t} \pi_{t}^{\top} \sqrt{\Sigma_{t}} d Z_{t}
$$


We consider an agent, endowed with a CRRA utility of terminal wealth with RRA coefficient $1-\gamma$ greater than zero, who wants to find the optimal allocation strategy $\left(\pi_{t}\right)$ which solves the following problem

$$
J\left(0, X_{0}, \Sigma_{0}\right)=\sup _{\pi} \mathbb{E}\left[\frac{X_{T}^{\gamma}}{\gamma}\right]
$$

where the investment horizon $T$ is assumed to be fixed and the set of admissible strategies $\left(\pi_{t}\right)$ is defined in order to grant the regularity of the wealth process and some additional integrability involving the utility function (see e.g. He and Pearson 1991 for technical details).

Following the duality-based argument of He and Pearson (1991), the optimization problem splits into two steps: the first step is the difficult one and consists in finding the optimal value function, while the second step is quite standard and consists in finding the optimal investment strategy by a martingale representation argument.

The following proposition represents the solution of the first step procedure:

Proposition 3.1. If the dynamic of the covariance matrix is given by (1) then the value function of the problem (11) is given by:

$$
J\left(0, X_{0}, \Sigma_{0}\right)=\frac{X_{0}^{\gamma}}{\gamma} e^{(1-\gamma) \operatorname{Tr}\left[A(0, T) \Sigma_{0}\right]+(1-\gamma) B(0, T)}
$$

where the deterministic matrix $A(t, T)$ (resp. function $B(t, T)$ ) is explicitly given by (42) (resp. by (43)) in the Appendix.

Now by a standard argument we can find the optimal investment strategy: this is given by the following proposition.

Proposition 3.2. The optimal portfolio solution associated to the problem (11) is given by

$$
\pi_{t}=\frac{\lambda_{0}}{1-\gamma}+2 A Q^{\top} \rho,
$$

where $A$ is explicitly given by (42) in the Appendix.

Remark 3.1. Taking $n=2$ and $\rho=(1,0)^{\top}$ we obtain the solution found in Buraschi et al. (2006)

$$
\pi=\frac{1}{1-\gamma}\left(\begin{array}{c}
\lambda_{0}^{1} \\
\lambda_{0}^{2}
\end{array}\right)+2\left(\begin{array}{c}
A_{11} Q_{11}+A_{12} Q_{12} \\
A_{21} Q_{11}+A_{22} Q_{12}
\end{array}\right),
$$

Remark 3.2. In the 2 -dimensional case with $\rho^{\top} \rho<1$ we obtain the general solution

$$
\begin{aligned}
\pi_{t}^{*}= & \frac{1}{1-\gamma}\left(\left(\begin{array}{c}
\lambda_{0}^{1} \\
\lambda_{0}^{2}
\end{array}\right)-\sqrt{1-\rho_{1}^{2}-\rho_{2}^{2}}\left(\begin{array}{c}
l^{1} \\
l^{2}
\end{array}\right)\right) \\
& +2\left(\begin{array}{c}
A_{11}\left(Q_{11} \rho_{1}+Q_{21} \rho_{2}\right)+A_{12}\left(Q_{12} \rho_{1}+Q_{22} \rho_{2}\right) \\
A_{21}\left(Q_{11} \rho_{1}+Q_{21} \rho_{2}\right)+A_{22}\left(Q_{12} \rho_{1}+Q_{22} \rho_{2}\right)
\end{array}\right)
\end{aligned}
$$


Remark 3.3. The optimal strategy in the incomplete market can also be deduced from the solution of the complete market case by constraining the optimal strategy to assign a zero proportion of wealth in the (co)variance swaps: this is the primal approach followed by Leippold et al. (2007).

In our empirical analysis we are going to show that, contrarily to what stated in Buraschi et al. (2006), the size of the intertemporal hedging demand is small in absolute value. In line of principle it may happen that the hedging demand is small due to some compensating effects on the sign of the variance-covariance components. Then, in the following proposition we decompose the hedging demand into pure volatility and co-volatility effects as in Buraschi et al. (2006), in order to show if the sizes of all these effects are indeed small. For simplicity and without loss of generality we illustrate the results in the two assets case.

Proposition 3.3. The optimal trading strategy due to the stochastic volatility can be decomposed in a correlation hedging component $\pi_{\text {corr }}$, covariation hedging component $\pi_{\text {cov }}$ and a volatility hedging component $\pi_{\text {vor }}$. Within the WASC model these quantities for the first asset are given by

$$
\begin{aligned}
\pi_{\text {vol }}^{1} & =2 A^{11}\left(Q^{\top} \rho\right)^{1} \\
\pi_{\text {covol }}^{1} & =2 A^{12} \bar{\rho} \sqrt{\frac{\Sigma^{22}}{\Sigma^{11}}}\left(Q^{\top} \rho\right)^{1} \\
\pi_{\text {corr }}^{1} & =2 A^{12}\left(-\bar{\rho} \sqrt{\frac{\Sigma^{22}}{\Sigma^{11}}}\left(Q^{\top} \rho\right)^{1}+\left(Q^{\top} \rho\right)^{2}\right)
\end{aligned}
$$

and for the second asset

$$
\begin{aligned}
\pi_{\text {vol }}^{2} & =2 A^{22}\left(Q^{\top} \rho\right)^{2} \\
\pi_{\text {covol }}^{2} & =2 A^{12} \bar{\rho} \sqrt{\frac{\Sigma^{11}}{\Sigma^{22}}}\left(Q^{\top} \rho\right)^{2} \\
\pi_{\text {corr }}^{2} & =2 A^{12}\left(-\bar{\rho} \sqrt{\frac{\Sigma^{11}}{\Sigma^{22}}}\left(Q^{\top} \rho\right)^{2}+\left(Q^{\top} \rho\right)^{1}\right)
\end{aligned}
$$

where $\left(Q^{\top} \rho\right)^{i}$ stands for the $i^{\text {th }}$ element of the vector $Q^{\top} \rho$ and $\bar{\rho}=\frac{\Sigma^{12}}{\sqrt{\Sigma^{11} \Sigma^{22}}}$.

\subsection{The portfolio problem in the completed market}

Now we consider the problem of an agent who invests an initial wealth $X_{0}$ into a the financial market consisting in the $n$ risky assets $S_{t}^{1}, . ., S_{t}^{n}$ but also in the $n(n+1) / 2$ (co)variance swaps described in the Section 2. Let us denote by $\left(\pi_{t}\right)=\left(\pi_{t}^{1}, \ldots, \pi_{t}^{n}\right)^{\top}$ the proportion of wealth $X_{t}$ invested into the risky assets $S_{t}^{1}, . ., S_{t}^{n}$, and by $\Pi_{t} \in$ $M_{n}$ the fraction of wealth invested as notional for the (co-)variance swap contract 
$(V S(t, T)-K)$, where at each time the agent initiates new variance swap contracts with the delivery price $K$ set to be $K=V S(t, T)$, so that the variance swap contract has zero initial value. This implies that under the risk neutral probability measure $\mathbb{Q}$ the dynamics of the variance swap contract is a martingale:

$$
\begin{aligned}
d V S(t, T)= & 0 d t+\frac{1}{T-t} \int_{t}^{T} e^{(u-t) M^{\mathbb{Q}}}\left(\sqrt{\Sigma_{t}}\left(d W_{t}-\sqrt{\Sigma_{t}} \Lambda d t\right) Q\right. \\
& \left.+Q^{\top}\left(d W_{t}-\sqrt{\Sigma_{t}} \Lambda d t\right)^{\top} \sqrt{\Sigma_{t}}\right) e^{(u-t) M^{\mathbb{Q}^{\top}}} d u .
\end{aligned}
$$

Remark 3.4. The dynamics of the matrix contract $V S(t, T)$ is symmetric. This symmetry can lead to some misleading interpretation of market incompleteness in our framework. In fact, the uncertainty induced by the (symmetric) stochastic volatility matrix $\Sigma_{t}$ is strictly smaller than the one generated by the $n^{2}$ Brownian motions $W$, so that the $n(n+1) / 2$ (co-)variance swaps are sufficient to complete the market. This is a wellknown feature of the symmetric matrix representation of the Wishart process, which carries less information than the whole filtration generated by the sources of uncertainty (see Gourieroux and Sufana (2004), page 17). Roughly speaking, we improperly call market completeness the possibility to perfectly hedge any derivative contract written on the traded assets. In other words, we will not consider the (mathematically interesting) problem of pricing and hedging a contingent claim whose payoff may depend on each component of the sources of uncertainty $W, B$ (see the paper of Davis and Obloj (2007) for some recent results of this approach in a Heston framework).

By using the previous dynamics, the (self-financing) wealth process evolves according to the following SDE (see also Leippold et al. (2007)):

$$
\frac{d X_{t}^{\pi, \Pi}}{X_{t}^{\pi, \Pi}}=\pi_{t}^{\top}\left(\Sigma_{t} \lambda_{0} d t+\sqrt{\Sigma_{t}} d Z_{t}\right)+\operatorname{Tr}\left[\Pi_{t} d V S(t, T)\right] .
$$

We consider the same optimization problem as in the incomplete case, i.e.:

$$
J\left(0, X_{0}, \Sigma_{0}\right)=\sup _{\pi, \Pi} \mathbb{E}\left[\frac{\left(X_{T}^{\pi, \Pi}\right)^{\gamma}}{\gamma}\right],
$$

where the set of admissible strategies $\left(\pi^{*}, \Pi^{*}\right)$ is defined in the usual way in order to insure the existence of a strong solution to the SDE satisfied by the wealth process. By the same argument as in the incomplete market case we get the following result:

Proposition 3.4. If the dynamic of the covariance matrix is given by (1) then the value function of problem (20) in the completed market is given by:

$$
J\left(0, X_{0}, \Sigma_{0}\right)=\frac{X_{0}^{\gamma}}{\gamma} e^{(1-\gamma) \operatorname{Tr}\left[A(0, T) \Sigma_{0}\right]+(1-\gamma) B(0, T)}
$$

where the deterministic matrix $A(t, T)$ (resp. function $B(t, T)$ ) is explicitly given by (47) (resp. by (48)) in the Appendix. 
Proposition 3.5. Suppose that $1-\rho^{\top} \rho \neq 0$. If the agent can invest in a complete market consisting in the risky assets and the (co-)variance swaps, then the optimal proportion of wealth invested into the risky assets is given by

$$
\begin{aligned}
\pi_{t} & =-\frac{1}{1-\rho^{\top} \rho} \frac{\partial_{X} J}{X_{t} \partial_{X X}^{2} J}\left(\lambda_{0}+\Lambda \rho\right), \\
& =\frac{1}{1-\rho^{\top} \rho} \frac{1}{1-\gamma}\left(\lambda_{0}+\Lambda \rho\right)
\end{aligned}
$$

while the optimal fractions of wealth invested as notional in (co-)variance swaps are implicitely given by

$$
\begin{aligned}
\frac{1}{\tau} \int_{0}^{\tau} e^{s M^{\mathbb{Q}^{\top}}} \Pi_{t} e^{s M^{\mathbb{Q}}} d s & =\frac{1}{4(\gamma-1)}\left(\Lambda\left(Q^{-1}\right)^{\top}+\left(Q^{-1}\right) \Lambda^{\top}\right) \\
& +\frac{1}{4(\gamma-1)}\left(\frac{l \rho^{\top}}{\sqrt{1-\rho^{\top} \rho}}\left(Q^{-1}\right)^{\top}+Q^{-1} \frac{\rho l^{\top}}{\sqrt{1-\rho^{\top} \rho}}\right)+A,
\end{aligned}
$$

where $A=A(t, T)$ is given by (47) in the Appendix.

Our result is analogous to the one obtained by Leippold et al. (2007) in the Heston framework. There is no intertemporal hedging demand for the risky assets in the presence of (co-)variance swap contracts: the optimal strategy is just the classic constant proportion solution found by Merton (1971). However, as we shall see in the next section, the proportion invested into the risky assets changes dramatically with respect to the incomplete market case. This is basically due to two reasons. The first one is linked to the high risk premium of (co)volatility risk $\Lambda$ estimated in the market, while the second relies on leverages effects due to the presence of (co)volatility swaps.

Remark 3.5. In Buraschi et al. (2006) $\rho^{\top} \rho=1$, so that they would find a degenerate trading strategy in the presence of variance swap contracts: here the presence of a Brownian motion orthogonal to the volatility noise is crucial. In other words, in the model of Buraschi et al. (2006), the presence in the market of the (co-)variance swaps makes the risky assets redundant.

\section{Empirical results}

In this section we present our empirical results when using the WASC model on a real dataset. We first briefly review the methodology developed to estimate the WASC model under the historical measure. We will basically follow Da Fonseca et al. (2007a) who estimated the WASC model using a C-GMM based procedure. Then, we focus on the estimation of the volatility risk premium, that is the risk premia associated to the matrix Wishart process. Using a Kalman filter based methodology as presented in Duffee and Stanton (2004) on a database of variance swaps (see also Leippold et al. (2007)) we estimate the variance risk premia, thus identifying the WASC model under both the historical probability measure and the risk neutral one. 


\subsection{Estimation of the WASC model under $\mathbb{P}$}

We briefly recall the main results of Da Fonseca et al. (2007a) on the estimation of the WASC under the historical probability. Based on the work of Chacko and Viceira (2005), we estimate the WASC model using moment conditions based on the empirical characteristic function. Methodologies of this kind are known as spectral generalized method of moments. This framework is particularly adapted to the WASC since its characteristic function is known in a closed form expression. Furthermore, using a continuum of moments as in Carrasco et al. (2007) increases the efficiency of the estimators when compared to those obtained in Chacko and Viceira (2005). The dataset used in the estimation consists in SP500 and DAX quotes ranging from January 2nd 1990 to June 30th 2007. We found that when the parameters are conveniently aggregated, the WASC model displays a behavior comparable to the one of the Heston model. What is more the estimated WASC models reproduce stylized effects such as contagion effects that can not be handled within the Heston model. Table 1 provides the parameter estimates for the WASC model with our dataset. We refer to Da Fonseca et al. (2007a) for further details on the methodology and on the financial interpretation of the estimated parameters.

\subsection{Estimation of the volatility risk premium}

We turn our attention to the estimation of the variance risk premium. We use a database of variance swaps quotes on the indexes SP500, DAX ranging from June 21st 2006 to June 23rd 2007. Each day we have a term structure of variance swaps with maturities ranging from 1 month to 21 months, which makes 21 contracts.

The risk neutral dynamics of the (co-)variance matrix is:

$$
d \Sigma_{t}=\left(\Omega \Omega^{\top}+\left(M+Q^{\top} \Lambda^{\top}\right) \Sigma_{t}+\Sigma_{t}\left(M+Q^{\top} \Lambda^{\top}\right)^{\top}\right) d t+\sqrt{\Sigma_{t}} d W_{t}^{\mathbb{Q}} Q+Q^{\top}\left(d W_{t}^{\mathbb{Q}}\right)^{\top} \sqrt{\Sigma_{t}} .
$$

Pricing (co)variance swaps contracts involves the computation of

$$
\frac{1}{\tau} \int_{t}^{t+\tau} E_{t}^{\mathbb{Q}}\left[\Sigma_{u}\right] d u
$$

Using the dynamics of the Wishart process and denoting by $X_{u}=E_{t}^{\mathbb{Q}}\left[\Sigma_{u}\right]$, we deduce that

$$
\frac{d X_{u}}{d u}=\Omega \Omega^{\top}+\left(M+Q^{\top} \Lambda^{\top}\right) X_{u}+X_{u}\left(M+Q^{\top} \Lambda^{\top}\right)^{\top} .
$$

In order to compute the (co)-variance swaps value it is more convenient to rewrite the dynamics of equation (24) in a vector form. Denoting $\bar{X}_{u}=V e c\left(X_{u}\right)=\left(X_{u}^{11}, X_{u}^{21}, \ldots, X_{u}^{n n}\right)^{\top}$ and $\bar{B}=V e c\left(\Omega \Omega^{\top}\right)$, we are lead to the system:

$$
\frac{d \bar{X}_{u}}{d u}=\bar{A} \bar{X}_{u}+\bar{B}
$$


with $\bar{A}=I \otimes\left(M+Q^{\top} \Lambda^{\top}\right)+\left(M+Q^{\top} \Lambda^{\top}\right) \otimes I^{4}$. Simple computations give

$$
\frac{1}{\tau} \int_{t}^{t+\tau} \bar{X}_{u} d u=\frac{\bar{A}^{-1}}{\tau}\left[e^{\tau \bar{A}}-I\right] \bar{X}_{t}-\bar{A}^{-1} \bar{B}+\frac{1}{\tau}\left[e^{\tau \bar{A}}-I\right]\left(\bar{A}^{-1}\right)^{2} \bar{B} .
$$

As a consequence the (co)-variance swap contracts are affine transform of the Wishart process and can be written as

$$
V S(t, T)=\tilde{A} \bar{X}_{t}+\tilde{B}
$$

with $\tilde{A}$ and $\tilde{B}$ identified from equation (26). We observe an affine transform of the latent factor $X_{t}$ which leads to estimation strategies similar to those currently developed for affine term structure models. Since we have a factor model for the (co)-variance swaps contracts, we can duplicate the (co)variance swap contract using variance swaps contracts with different maturities. From the estimation point of view, we chose the (linearized) Kalman filtering estimation strategy as in Duffee and Stanton (2004) and Leippold et al. (2007). In order to fit within usual notations we rewrite the dynamics of the Wishart under the historical probability in vector form. Denoting $Z_{t}=\left(\Sigma_{t}^{11}, \Sigma_{t}^{12}, \Sigma_{t}^{22}\right)^{\top}$ it is straightforward to obtain

$$
d Z_{t}=\left(L Z_{t}+K\right) d t+\Theta\left(Z_{t}\right) d \bar{W}_{t}
$$

where $\bar{W}_{t}=\left(W_{t}^{11}, W_{t}^{12}, W_{t}^{21}, W_{t}^{22}\right)^{\top}$ and $K, L, \Theta(Z)$ suitably defined from the WASC dynamics. Using Kalman filtering theory as presented in Brandt and He (2006) and Duffee and Stanton (2004) from the system (27) and (28) we estimate the market price of risk $\Lambda$ of the volatility. Table 2 presents the estimates of the market price of volatility risk. Table 3 describes the descriptive statistics around the dataset, while Table 4 provides descriptive statistics about the obtained variance swaps pricing errors.

\subsection{Analysis of the estimation results}

In this section, we review the main estimation results of the paper. We first discuss the empirical properties of the dataset and of the estimated variance swaps dynamics. Then we turn our attention toward the analysis of the hedging policy yielded by the estimated WASC model for both the complete and incomplete cases.

Summary statistics for the variance swap dataset are provided in Table 4. This dataset presents the usual features of variance time series, including asymmetric and leptokurtic distributions and a high degree of autocorrelation. The variance swap time series are presented in the Figures 2 for the DAX and 1 for the SP500. Most of the time, the term structure of variance swaps is increasing, but for a very special period of the dataset: around May 15th 2007, the launching of take profits in both the DAX and SP500 markets led to a drop in these indexes followed by an increase in the short term

\footnotetext{
${ }^{4} \otimes$ stands for the Kronecker product.
} 
volatility due to well known leverage effects.

The estimation results for the variance risk premia is provided in Table 2. The matrix risk correction presents values that are close in terms of sign and magnitude to those obtained in the univariate case by Leippold et al. (2007). The risk neutral matrix drift is definite negative, as expected. The Table 4 presents descriptive statistics around the pricing errors obtained with the estimated WASC model. Globally, the pricing errors are below one volatility percentage point for the whole time series studied. The pricing errors for the DAX are presented in Figure 4 and for the SP500 in Figure 3. As expected, the pricing errors get higher in may 2007, due to the aforementioned takeprofit event in the market. The quality of the variance swap pricing errors can also be viewed through the $R^{2}$ measure: the obtained figures are very close to the robust ones obtained in Leippold et al. (2007).

\subsection{Analysis of optimal strategies in incomplete and completed mar- ket}

In this subsection, we investigate the behavior of the optimal allocation strategies in both the incomplete and completed markets cases.

\subsubsection{The optimal strategy in the incomplete market}

When implementing the optimal solution for the WASC model using our dataset, we find that the size of the intertemporal hedging demand is indeed small (about $0.63 \%$ and 0.89\%), in accordance to Chacko and Viceira (2005) and Liu (2005). This is in contrast with the result of Buraschi et al. (2006), who declared a size of about $10 \%$ and $11 \%$. However, when implementing their strategy (using their model, estimates and dataset) we found a completely different result (around 0.7\% - 1.01\%): the re-computed results are in line with the one obtained in literature and in the WASC model. Thus, there seems to be a numerical mistake in their paper. Table 5 summarizes the aggregate hedging demands in (declared and correct) Buraschi et al. (2006) case as well as in our WASC case.

The main message in Buraschi et al. (2006) was that "the optimal demand to hedge correlation risk is a non-negligible fraction of the myopic portfolio, which often dominates the pure volatility hedging demand" (see panel B of table III in Buraschi et al. (2006)). When recomputing their solution, the domination of the correlation hedge vanishes.

The Table 9 presents the loadings for both stocks in the optimal portfolio computed in the incomplete market case: the sign is in line with the classic Merton solution, with two long positions. What is more, when increasing the volatility of the matrix 
covariance process, the hedging demand does not exhibit a strong sensitivity. This is a serious problem when dealing with risk measures.

\subsubsection{The optimal strategy in the completed market}

In the completed market, the hedging strategy is significantly different. The size of the hedging ratios on the stocks are large when compared with the incomplete market case. What is more, they mainly depend on the market price of volatility risk. Furthermore, the position on the stocks are of opposite sign, even though the market price of risk are both positive. This is basically due to the possibility of leverage effects. In fact, with respect to the (co)variance swaps we get similar conclusions: we end up with large hedging ratios in both assets and covariance swaps (in absolute value term) thus showing strong leverage effects. Our results are consistent with those obtained by Leippold et al. (2007).

A sensivity analysis with respect to volatility of volatility (the matrix $Q$ ) reported in Table 10 shows the strong dependence of the hedging ratios on this parameter. This should be compared with the incomplete market case where hedging ratios are only slightly altered. Thus the (co)variance risk can not be analyzed within the incomplete market, or more precisely a market of stocks only.

This confirms, if necessary, that (co)variance risk should be handled with assets depending on volatility. Obviously, the (co)variance swaps suit perfectly that objective. Any vanilla options would also be a convenient choice (see e.g. Hull (2008)), unlike stocks. The choice of variance swap obviously relies on the linearity of the payoff function.

Clearly, we need a new measure for (co)variance risk. We propose to set the problem in terms of additional utility: we compare the required initial endowment to reach the same expected utility in the completed and incomplete market cases. We associate the spread between these amount of wealth to the size of (co)variance risk. This criterion is related to the utility-based indifference pricing, the main differences being that we consider variance swap prices as given and our problem is set in a dynamic perspective since we allow for trading in this asset. Table 10 confirms that our risk measure is a suitable candidate for representing the (co)variance risk in financial markets. In fact, this measure is highly sensible to risk parameters, like volatility of volatility. From a quantitative point of view, our results indicate that in the incomplete market case, this initial wealth should be equal to about 250 times the corresponding endowment when variance swaps are available. 


\section{Conclusion}

In this paper, we propose a new (co)variance risk measure obtained when completing the stock market with non-redundant volatility products, i.e. variance swaps. We provide explicit formulas for the optimal portfolio in both the incomplete and completed market cases in a multivariate stochastic correlation framework. Once the model is estimated using the Kalman filter, an investigation of the empirical results obtained with SP500 and DAX clearly validates our approach.

\section{Appendix}

\section{1 (Co) Variance Swaps rates}

Using the dynamics of the Wishart process and denoting by $X_{u}=E_{t}^{\mathbb{Q}}\left[\Sigma_{u}\right]$, we deduce that

$$
\frac{d X_{u}}{d u}=\Omega \Omega^{\top}+M^{\mathbb{Q}} X_{u}+X_{u} M^{\mathbb{Q}^{\top}} .
$$

Set $X_{u}=Z_{u}^{-1} Y_{u}$ thus getting

$$
\begin{aligned}
& \frac{d}{d u}\left(\begin{array}{cc}
Y & Z
\end{array}\right)=\left(\begin{array}{ll}
Y & Z
\end{array}\right)\left(\begin{array}{cc}
M^{\mathbb{Q}^{\top}} & 0 \\
\Omega \Omega^{\top} & -M^{\mathbb{Q}}
\end{array}\right) \\
&\left(\begin{array}{ll}
Y_{u} & Z_{u}
\end{array}\right)=\left(\begin{array}{ll}
Y_{t} & Z_{t}
\end{array}\right) \exp (u-t)\left(\begin{array}{cc}
M^{\mathbb{Q}^{\top}} & 0 \\
\Omega \Omega^{\top} & -M^{\mathbb{Q}}
\end{array}\right) \\
&=\left(\begin{array}{ll}
Y_{t} & Z_{t}
\end{array}\right)\left(\begin{array}{cc}
A_{11} & A_{12} \\
A_{21} & A_{22}
\end{array}\right) \\
&=\left(\begin{array}{ll}
Y_{t} & Z_{t}
\end{array}\right)\left(\begin{array}{cc}
e^{(u-t) M^{\mathbb{Q}^{\top}}} & 0 \\
A_{21} & e^{-(u-t) M^{\mathbb{Q}}}
\end{array}\right),
\end{aligned}
$$

so that

$$
X_{u}=e^{(u-t) M^{\mathbb{Q}}} X_{t} e^{(u-t) M^{\mathbb{Q}^{\top}}}+e^{(u-t) M^{\mathbb{Q}}} A_{21},
$$

where we used the fact that $A_{12}=0$ and $\left(e^{-(u-t) M^{\mathbb{Q}}}\right)^{-1}=e^{(u-t) M^{\mathbb{Q}}}$.

By simple integration we obtain the matrix (co-)variance swap rate:

$$
\begin{aligned}
V S(t, T) & =\frac{1}{T-t} \int_{t}^{T} E_{t}^{\mathbb{Q}}\left[\Sigma_{u}\right] d u \\
& =\frac{1}{T-t} \int_{t}^{T} e^{(u-t) M^{\mathbb{Q}}} \Sigma_{t} e^{(u-t) M^{\mathbb{Q}}} d u+\frac{1}{T-t} \int_{t}^{T} e^{(u-t) M^{\mathbb{Q}}} A_{21} d u .
\end{aligned}
$$

Now from

$$
\begin{aligned}
V S^{i j}(t, T) & =\frac{1}{T-t} E_{t}^{\mathbb{Q}}\left[\int_{t}^{T} \Sigma_{u}^{i j} d u\right] \\
& =\frac{1}{T-t} E_{t}^{\mathbb{Q}}\left[\int_{t}^{T} \operatorname{Tr}\left[e_{i j} \Sigma_{u}\right] d u\right] \\
& =\frac{1}{T-t} \operatorname{Tr}\left[\int_{t}^{T} e_{i j} E_{t}^{\mathbb{Q}}\left[\Sigma_{u}\right] d u\right]
\end{aligned}
$$


we easily deduce

$$
V S^{i j}(t, T)=\frac{1}{T-t} \operatorname{Tr}\left[\Sigma_{t} \int_{t}^{T} L_{u}^{i j} d u\right]+\frac{1}{T-t} \int_{t}^{T} \operatorname{Tr}\left[e_{i j} e^{(u-t) M^{\mathbb{Q}}} A_{21} d u\right],
$$

where $L_{u}^{i j}=e^{(u-t) M^{\mathbb{Q}}} e_{i j} e^{(u-t) M^{\mathbb{Q}}}$.

\subsection{Proof of proposition 3.1}

The proof of proposition 3.1 is quite technical, so first of all we will give an idea of the procedure.

Following He and Pearson (1991), the dynamic optimization problem in the incomplete market can be transformed into a static one by duality theory, where the dual problem consists in finding the minimax martingale measure associated to the following problem:

$$
\begin{aligned}
J\left(0, X_{0}, \Sigma_{0}\right)= & \inf _{\nu \in Z^{\perp}} \sup _{X_{T}} \mathbb{E}\left[\frac{X_{T}^{\gamma}}{\gamma}\right] \\
& \text { s.to: } \mathbb{E}\left[H_{T} X_{T}\right] \leq X_{0}
\end{aligned}
$$

where $\nu$ lies in the orthogonal space with respect to the market price of risk spanned by the asset prices and it is formally introduced in He and Pearson (1991). The economic intuition of the previous problem is that in this incomplete market there are infinitely many price deflators, each of which can be written as the sum of two orthogonal components: the first one is associated to $\lambda_{0}$ which is spanned by the market, while the second one is parametrized by $\nu \in Z^{\perp}$, where (roughly speaking) $Z^{\perp}$ denotes the orthogonal part of the assets' noises. The minimax martingale measure corresponds to the state price density which minimizes this orthogonal part consistently with the CRRA utility criterion. The technical point consits in finding a suitable decomposition of the orthogonal part $Z^{\perp}$ in order to solve explicitely the minimization problem associated to the variable $\nu$.

Without loss of generality we will assume that $\rho^{\top} \rho \neq 0$. Notice that the case $\rho^{\top} \rho=0$ is easy to treat: it corresponds to the case where the volatility matrix is independent of assets' returns, and is well know that the optimal investment strategy in this case coincides with the Merton's ones, see e.g. Karatzas and Shreve (1998) page 305.

From a standard duality argument (see He and Person 1991) the optimal terminal wealth can be written in the following form:

$$
X_{T}^{*}=\left(y H_{T}\right)^{\frac{1}{\gamma-1}}
$$

so that

$$
J\left(0, X_{0}, \Sigma_{0}\right)=X_{0}^{\gamma} \inf _{\nu \in Z^{\perp}} \frac{1}{\gamma} \mathbb{E}\left[\left(H_{T}\right)^{\frac{\gamma}{\gamma-1}}\right]^{1-\gamma},
$$


therefore we can reduce to the following problem:

$$
\widehat{J}\left(0, Y_{0}, \Sigma_{0}\right)=\inf _{\nu \in Z^{\perp}} \mathbb{E}\left[\left(H_{T}\right)^{\frac{\gamma}{\gamma-1}}\right],
$$

where the state variables consist in the Wishart process $\Sigma$ and market returns $Y_{t}$ whose dynamics come directly from $(2)$.

Knowing that $d Z=\sqrt{1-\rho^{\top} \rho} d B+d W \rho$ we define $\left\{\alpha_{i} ; i=1 \ldots n-1\right\}$ such that

$$
\begin{array}{r}
\alpha_{i}^{\top} \alpha_{j}=\delta_{i j} \\
\alpha_{i}^{\top} \rho=0
\end{array}
$$

thus we have $I_{n}=\frac{\rho \rho^{\top}}{\rho^{\top} \rho}+\sum_{i=1}^{n-1} \alpha_{i} \alpha_{i}^{\top}$, where $I_{n}$ denotes the $n \times n$ identity matrix. Define

$$
\begin{aligned}
d Z_{i}^{\perp} & =d W \alpha_{i} i=1 \ldots n-1 \\
d Z_{n}^{\perp} & =-\sqrt{\rho^{\top} \rho} d B+\sqrt{\frac{1-\rho^{\top} \rho}{\rho^{\top} \rho}} d W \rho
\end{aligned}
$$

then $\left(Z, Z_{i}^{\perp}\right)$ are $n+1$ independent $n$ dimensional Brownian motions. Let $\left\{\nu_{i}=\right.$ $\left.\Lambda \alpha_{i} i=1 . . n-1\right\}$ be the projection of the maket price of volatility risk on the set of all possible directions $\alpha_{i}$ orthogonal to the market (and the Brownian motion $B$ ) and $\nu_{n}=\sqrt{\frac{1-\rho^{\top} \rho}{\rho^{\top} \rho}} \Lambda \rho+\sqrt{\rho^{\top} \rho}$, that is (minus) the projection of the risk premium $(-\Lambda \vdots l) \in M_{n \times(n+1)}$ along the direction $\left(\sqrt{\frac{1-\rho^{\top} \rho}{\rho^{\top} \rho}} \rho^{\top},-\sqrt{\rho^{\top} \rho}\right)$.

By standard techniques, let us consider the change of measure associated to the following Radon-Nykodim derivative:

$$
\frac{d \mathbb{P}^{\gamma}}{d \mathbb{P}} \quad \mid \quad \mathcal{F}_{t}=\mathcal{E}\left(\frac{\gamma}{\gamma-1} \sqrt{\Sigma} \lambda_{0}, Z\right)_{t} \prod_{i=1}^{n} \mathcal{E}\left(-\frac{\gamma}{\gamma-1} \sqrt{\Sigma} \nu_{i}, Z_{i}^{\perp}\right)_{t}
$$

where $\mathcal{E}(\theta, Z)_{t}=e^{\int_{0}^{t} \theta_{s}^{\top} d Z_{s}-\frac{1}{2} \int_{0}^{t} \theta_{s}^{\top} \theta_{s} d s}$. Under $\mathbb{P}^{\gamma}$ the Brownian motions change according to:

$$
\begin{aligned}
d B^{\gamma} & =d B+\frac{\gamma}{\gamma-1} \sqrt{\Sigma_{t}} l d t \\
d W_{t}^{\gamma} & =d W_{t}-\frac{\gamma}{\gamma-1} \sqrt{\Sigma_{t}} \Lambda d t \\
& =d W_{t}-\frac{\gamma}{\gamma-1} \sqrt{\Sigma_{t}}\left(\frac{\Lambda \rho \rho^{\top}}{\rho^{\top} \rho}+\sum_{i=1}^{n-1} \nu_{i} \alpha_{i}^{\top}\right) d t
\end{aligned}
$$

and from (8) and the definition of $\nu_{n}$ it follows that

$$
\Lambda \rho=\nu_{n} \sqrt{\rho^{\top} \rho\left(1-\rho^{\top} \rho\right)}-\lambda_{0} \rho^{\top} \rho
$$


so that

$$
d W_{t}^{\gamma}=d W_{t}-\frac{\gamma}{\gamma-1} \sqrt{\Sigma_{t}}\left(\sqrt{\frac{1-\rho^{\top} \rho}{\rho^{\top} \rho}} \nu_{n} \rho^{\top}+\sum_{i=1}^{n-1} \nu_{i} \alpha_{i}^{\top}-\lambda_{0} \rho^{\top}\right) d t .
$$

The orthogonal decomposition for the Brownian motions reads:

$$
\begin{aligned}
d Z^{\gamma} & =d Z+\frac{\gamma}{\gamma-1} \sqrt{\Sigma} \lambda_{0} d t \\
d\left(Z_{i}^{\gamma}\right)^{\perp} & =d Z_{i}^{\perp}-\frac{\gamma}{\gamma-1} \sqrt{\Sigma} \nu_{i} d t
\end{aligned}
$$

such that under $\mathbb{P}^{\gamma}$ the dynamics of the Wishart covariance matrix becomes

$$
d \Sigma_{t}=\left(\Omega \Omega^{\top}+M^{\gamma} \Sigma_{t}+\Sigma_{t}\left(M^{\gamma}\right)^{\top}\right) d t+\sqrt{\Sigma_{t}} d W_{t}^{\gamma} Q+Q^{\top}\left(d W_{t}^{\gamma}\right)^{\top} \sqrt{\Sigma_{t}},
$$

with

$$
M^{\gamma}=M+\frac{\gamma}{\gamma-1} Q^{\top}\left(\sqrt{\frac{1-\rho^{\top} \rho}{\rho^{\top} \rho}} \nu_{n} \rho^{\top}+\sum_{i=1}^{n-1} \nu_{i} \alpha_{i}^{\top}-\lambda_{0} \rho^{\top}\right)^{\top}
$$

while the dynamics of the market returns become

$$
d Y_{t}=\left(r-\frac{1}{2} \operatorname{Vec}\left(e_{i i} \Sigma_{t}\right)-\frac{1}{\gamma-1} \Sigma_{t} \lambda_{0}\right) d t+\sqrt{\Sigma_{t}} d Z_{t}^{\gamma} .
$$

With this change of measure the previous problem (32) becomes:

$$
\widehat{J}\left(0, Y_{0}, \Sigma_{0}\right)=\inf _{\nu} \mathbb{E}^{\gamma}\left[\exp \left\{-\frac{\gamma}{\gamma-1} \int_{0}^{T} r d s+\frac{\gamma}{2(\gamma-1)^{2}} \int_{0}^{T}\left(\lambda_{0}^{\top} \Sigma_{s} \lambda_{0}+\sum_{i=1}^{n} \nu_{i}^{\top} \Sigma_{s} \nu_{i}\right) d s\right\}\right] .
$$

By the Feynman-Kac formula $\widehat{J}\left(0, Y_{0}, \Sigma_{0}\right)$ solves the following (HJB) PDE:

$$
\begin{aligned}
-\frac{\partial \widehat{J}}{\partial t} & =\inf _{\nu_{i}, i=1, . ., n}\left\{\mathcal{L}_{Y, \Sigma}^{\gamma} \widehat{J}+\widehat{J}\left(-\frac{\gamma}{\gamma-1} r+\frac{\gamma}{2(\gamma-1)^{2}}\left(\lambda_{0}^{\top} \Sigma \lambda_{0}+\sum_{i=1}^{n} \nu_{i}^{\top} \Sigma \nu_{i}\right)\right)\right\} \\
\widehat{J}(T, Y, \Sigma) & =1
\end{aligned}
$$

where

$$
\begin{aligned}
\mathcal{L}_{Y, \Sigma}^{\gamma} & =\operatorname{Tr}\left[\Omega \Omega^{\top} D+M^{\gamma} \Sigma D+\Sigma\left(M^{\gamma}\right)^{\top} D+2 \Sigma D Q^{\top} Q D\right] \\
& +\nabla_{Y}\left(r \mathbf{1}-\frac{1}{2} V e c\left[\Sigma^{i i}\right]-\frac{1}{\gamma-1} \Sigma \lambda_{0}\right)+\frac{1}{2} \nabla_{Y} \Sigma \nabla_{Y}^{\top} \\
& +2 \operatorname{Tr}\left[D Q^{\top} \rho \nabla_{Y} \Sigma\right]
\end{aligned}
$$

denotes the infinitesimal generator of the Wishart dynamics together with the assets' returns under the measure $\mathbb{P}^{\gamma}$.

Let us now find out the optimal values for $\nu_{i}^{*}, i=1, . ., n$ associated to the minimax probability measure. The first order conditions give 


$$
0=\partial_{\nu_{i}} \mathcal{L}_{Y, \Sigma}^{\gamma} \widehat{J}+\widehat{J} \frac{\gamma}{2(\gamma-1)^{2}} \partial_{\nu_{i}}\left(\nu_{i}^{\top} \Sigma \nu_{i}\right)
$$

then

$$
\begin{aligned}
\nu_{i}^{*} & =(1-\gamma)\left(\frac{D \widehat{J}+D \widehat{J}^{\top}}{\widehat{J}}\right) Q^{\top} \alpha_{i} \quad i=1 \ldots n-1 \\
\nu_{n}^{*} & =(1-\gamma)\left(\frac{D \widehat{J}+D \widehat{J}^{\top}}{\widehat{J}}\right) \sqrt{\frac{1-\rho^{\top} \rho}{\rho^{\top} \rho}} Q^{\top} \rho .
\end{aligned}
$$

By replacing $\nu^{*}$ into the HJB equation and using the matrix identity decomposition based on $\left\{\alpha_{i} ; i=1 \ldots n-1\right\}$ we obtain:

$$
\begin{aligned}
-\partial_{t} \widehat{J} & =\operatorname{Tr}\left[\Omega \Omega^{\top} D \widehat{J}+M \Sigma D \widehat{J}+\Sigma M^{\top} D \widehat{J}\right]+\operatorname{Tr}\left[2 \Sigma D Q^{\top} Q D \widehat{J}\right]-\widehat{J} \frac{\gamma}{\gamma-1} r \\
& +\widehat{J} \frac{\gamma}{2(\gamma-1)^{2}} \lambda_{0}^{\top} \Sigma \lambda_{0}+2 \operatorname{Tr}\left[D Q^{\top} \rho \nabla_{Y} \Sigma \widehat{J}\right] \\
& +\nabla_{Y}\left(r \mathbf{1}-\frac{1}{2} V e c\left[\Sigma^{i i}\right]-\frac{1}{\gamma-1} \Sigma \lambda_{0}\right) \widehat{J}+\frac{1}{2} \nabla_{Y} \Sigma \nabla_{Y}^{\top} \widehat{J} \\
& -\operatorname{Tr}\left[\frac{\gamma}{2} \widehat{J} \Sigma\left(\frac{D \widehat{J}}{\widehat{J}}+\frac{D \widehat{J}^{\top}}{\widehat{J}}\right) Q^{\top}\left(I_{n}-\frac{\rho \rho^{\top}}{\rho^{\top} \rho}\right) Q\left(\frac{D \widehat{J}}{\widehat{J}}+\frac{D \widehat{J}^{\top}}{\widehat{J}}\right)\right] \\
& -\operatorname{Tr}\left[\frac{\gamma}{2} \widehat{J} \Sigma\left(\frac{D \widehat{J}}{\widehat{J}}+\frac{D \widehat{J}^{\top}}{\widehat{J}}\right) Q^{\top} \rho \rho^{\top} Q\left(\frac{D \widehat{J}}{\widehat{J}}+\frac{D \widehat{J}^{\top}}{\widehat{J}}\right)\right]\left(\frac{1-\rho^{\top} \rho}{\rho^{\top} \rho}\right) \\
& -\frac{\gamma}{\gamma-1} \operatorname{Tr}\left[Q^{\top} \rho \lambda_{0}^{\top} \Sigma D \widehat{J}+\lambda_{0} \rho^{\top} Q D \widehat{J} \Sigma\right] \\
\widehat{J}(T, Y, \Sigma) & =1 .
\end{aligned}
$$

We guess an exponential affine function of the form

$$
\widehat{J}(t, Y, \Sigma)=e^{\operatorname{Tr}[A(t, T) \Sigma]+B(t, T)+C(t, T)^{\top} Y},
$$

so that

$$
\begin{aligned}
\partial_{t} \widehat{J} & =\widehat{J} \operatorname{Tr}\left[\partial_{t} A(t, T) \Sigma\right]+\widehat{J} \partial_{t} B(t, T)+\widehat{J} \partial_{t} C(t, T)^{\top} Y \\
D \widehat{J} & =\widehat{J} A(t, T) .
\end{aligned}
$$

Plugging it into the HJB equation and collecting terms gives the matrix Riccati ODE:

$$
\begin{aligned}
-\partial_{t} A & =A\left(M-\frac{\gamma}{\gamma-1} Q^{\top} \rho \lambda_{0}^{\top}+Q^{\top} \rho C^{\top}\right)+\left(M-\frac{\gamma}{\gamma-1} Q^{\top} \rho \lambda_{0}^{\top}+Q^{\top} \rho C^{\top}\right)^{\top} A \\
& +2 A Q^{\top} Q A-2 \gamma A Q^{\top}\left(I_{n}-\frac{\rho \rho^{\top}}{\rho^{\top} \rho}\right) Q A+\frac{\gamma}{2(\gamma-1)^{2}} \lambda_{0} \lambda_{0}^{\top} \\
& +\frac{1}{2} C C^{\top}-\frac{1}{2} C_{i} e_{i i}-\frac{1}{\gamma-1} C \lambda_{0}^{\top}-2 \gamma A Q^{\top} \rho \rho^{\top} Q A\left(\frac{1-\rho^{\top} \rho}{\rho^{\top} \rho}\right)
\end{aligned}
$$


and the following linear ODEs for $B(t, T)$ and $C(t, T)$ :

$$
\begin{aligned}
& -\partial_{t} B=\operatorname{Tr}\left[\Omega \Omega^{\top} A\right]+C^{\top} r \mathbf{1}-\frac{\gamma}{\gamma-1} r \\
& -\partial_{t} C=0
\end{aligned}
$$

the initial conditions being $A(T, T)=0 \in M_{n}, B(T, T)=0$ and $C(T, T)=0 \in \mathbb{R}^{n}$. We deduce that $C(t, T) \equiv 0$ therefore (40) can be written in a simpler form

$$
\left\{\begin{array}{l}
-\partial_{t} A=A \Gamma+\Gamma^{\top} A+A \Delta A+K \\
A(T, T)=0
\end{array}\right.
$$

with

$$
\begin{aligned}
\Gamma & =M-\frac{\gamma}{\gamma-1} Q^{\top} \rho \lambda_{0}^{\top}, \\
\Delta & =2 Q^{\top}\left(I_{n}(1-\gamma)+\gamma \rho \rho^{\top}\right) Q \\
K & =\frac{\gamma}{2(\gamma-1)^{2}} \lambda_{0} \lambda_{0}^{\top} .
\end{aligned}
$$

Using the linearization procedure we obtain:

$$
\begin{aligned}
A(t, T) & =P_{22}^{-1} P_{21} \\
B(t, T) & =-\frac{\beta}{2} \operatorname{Tr}\left[\log P_{22}+(T-t) \Gamma\right]-\frac{\gamma(T-t) r}{\gamma-1},
\end{aligned}
$$

with

$$
\left(\begin{array}{ll}
P_{11} & P_{12} \\
P_{21} & P_{22}
\end{array}\right)=\exp \left((T-t)\left(\begin{array}{cc}
\Gamma & -\Delta \\
K & -\Gamma^{\top}
\end{array}\right)\right) .
$$

The last thing to check is the absence of duality gap, that is that the value function of the primal problem and the dual one are the same. From standard duality theory (see e.g. Hobson (2004)) it turns out that there is no duality gap if

$$
\mathbb{E}_{t}\left[\left(H_{T}\right)^{\frac{\gamma}{\gamma-1}}\right]<+\infty
$$

This is equivalent to say that the flow of the matrix Riccati ODE (41) is well defined, and by Grasselli and Tebaldi (2008) this happens provided that the quadratic term in the Riccati ODE is positive definite. Therefore there is no duality gap if

$$
I_{n}(1-\gamma)+\gamma \rho \rho^{\top}>>0
$$

Now, for $\gamma<0$

$$
I_{n}(1-\gamma)+\gamma \rho \rho^{\top}=I_{n}-\gamma\left(I_{n}-\rho \rho^{\top}\right)
$$


is positive definite because $\gamma<0$ and $\left(I_{n}-\rho \rho^{\top}\right)$ is positive definite, in fact from

$$
\left(I_{n}-\rho \rho^{\top}\right)\left(I_{n}+\frac{\rho \rho^{\top}}{1-\rho^{\top} \rho}\right)=I_{n}
$$

it follows that it is the inverse of a positive definite matrix.

Otherwise, for $0<\gamma<1$,

$$
I_{n}-\gamma\left(I_{n}-\rho \rho^{\top}\right)>>I_{n}-\left(I_{n}-\rho \rho^{\top}\right),
$$

which is also positive definite, since $\rho \rho^{\top}$ is. The proof is now complete.

\subsection{Proof of proposition 3.2}

From the expression of the optimal terminal wealth (31) in terms of the price deflator and the fact that under optimality the product $X_{t}^{*} H_{t}$ is a martingale it follows that

$$
X_{t}=H_{t}^{\frac{1}{\gamma-1}} e^{\operatorname{Tr}\left[A(t, T) \Sigma_{t}\right]+B(t, T)} .
$$

Now we differentiate both sides and we equate the diffusions terms, thus obtaining:

$$
\begin{aligned}
\pi^{\top} \sqrt{\Sigma} d Z & =\frac{1}{\gamma-1}\left(\operatorname{Tr}\left[\Lambda^{\top} \sqrt{\Sigma_{t}} d W_{t}\right]-l^{\top} \sqrt{\Sigma_{t}} d B_{t}\right)+2 \operatorname{Tr}[Q A \sqrt{\Sigma} d W] \\
& =\operatorname{Tr}\left[\left(\frac{1}{\gamma-1} \Lambda+2 A Q^{\top}: \frac{-1}{\gamma-1} l\right)(d W: d B)^{\top} \sqrt{\Sigma}\right]
\end{aligned}
$$

Denoting by $\tilde{\rho}=\left(\rho^{\top}, \sqrt{1-\rho^{\top} \rho}\right)^{\top},\left\{u_{i}=\left(\alpha_{i}^{\top}, 0\right)^{\top} i=1 . . n-1\right\}$ and $u_{n}=\left(\frac{\sqrt{1-\rho^{\top} \rho}}{\sqrt{\rho^{\top} \rho}} \rho^{\top},-\sqrt{\rho^{\top} \rho}\right)^{\top}$ we have the identity decomposition

$$
I_{n+1}=\tilde{\rho} \tilde{\rho}^{\top}+\sum_{i=1}^{n} u_{i} u_{i}^{\top},
$$

so that the previous equality reads:

$$
\begin{aligned}
\operatorname{Tr}\left[\pi \tilde{\rho}^{\top}(d W: d B)^{\top} \sqrt{\Sigma}\right] & =\operatorname{Tr}\left[\left(\frac{1}{\gamma-1} \Lambda+2 A Q^{\top}: \frac{-1}{\gamma-1} l\right) I_{n+1}(d W \vdots d B)^{\top} \sqrt{\Sigma}\right] \\
& =\operatorname{Tr}\left[\left(\frac{1}{\gamma-1} \Lambda+2 A Q^{\top}: \frac{-1}{\gamma-1} l\right)\left(\tilde{\rho} \tilde{\rho}^{\top}+\sum_{i=1}^{n} u_{i} u_{i}^{\top}\right)(d W: d B)^{\top} \sqrt{\Sigma}\right]
\end{aligned}
$$

Now we identify the diffusion terms along the direction $\tilde{\rho}$ and we get:

$$
\begin{aligned}
\pi & =\frac{1}{\gamma-1} \Lambda \rho+2 A Q^{\top} \rho-\frac{1}{\gamma-1} l \sqrt{1-\rho^{\top} \rho} \\
& =\frac{\lambda_{0}}{1-\gamma}+2 A Q^{\top} \rho .
\end{aligned}
$$




\subsection{Proof of proposition 3.3}

The hedging component due to stochastic volatility is given by $2 A Q^{\top} \rho$ which is equal to $\frac{-2 J_{X \Sigma}}{J_{X X}} Q^{\top} \rho$ whose first row can be rewritten as

$$
\begin{array}{r}
-\frac{2 \partial_{X \Sigma^{11}} J}{X \partial_{X X}}\left(Q^{\top} \rho\right)^{1}-\frac{2 \partial_{X \Sigma^{12}} J}{X \partial_{X X}} \frac{\bar{\rho}}{2} \sqrt{\frac{\Sigma^{22}}{\Sigma^{11}}}\left(Q^{\top} \rho\right)^{1} \\
-\frac{2 \partial_{X \Sigma^{12}}}{X \partial_{X X}} J \sqrt{\Sigma^{11} \Sigma^{22}}\left(-\frac{\bar{\rho}}{2 \Sigma^{11}}\left(Q^{\top} \rho\right)^{1}+\frac{1}{\sqrt{\Sigma^{11} \Sigma^{22}}}\left(Q^{\top} \rho\right)^{2}\right)
\end{array}
$$

Following Buraschi et al. (2006) we qualify the first term as the pure volatility hedging component, the second term is the covariance hedging due to volatility and the last term is the correlation hedging component. Using the fact that $-\frac{\partial_{X \Sigma}^{2} J}{X \partial_{X X}^{2} J}=A$ we obtain the annonced result.

\subsection{Proof of Proposition 3.4}

By standard arguments based on duality theory in complete markets (see e.g. Karatzas and Shreve 1998), the value function $J\left(0, X_{0}, \Sigma_{0}\right)$ is given by

$$
J\left(0, X_{0}, \Sigma_{0}\right)=\frac{X_{0}^{\gamma}}{\gamma} \mathbb{E}\left[\left(H_{T}\right)^{\frac{\gamma}{\gamma-1}}\right]^{1-\gamma} .
$$

From (4),

$$
\begin{aligned}
\mathbb{E}\left[\left(H_{T}\right)^{\frac{\gamma}{\gamma-1}}\right]= & \mathbb{E}\left[\operatorname { e x p } \left(-\frac{\gamma}{\gamma-1} r T-\frac{\gamma}{2(\gamma-1)} \operatorname{Tr}\left[\int_{0}^{T} \Lambda^{\top} \Sigma_{s} \Lambda d s\right]-\frac{\gamma}{2(\gamma-1)} \int_{0}^{T} l^{\top} \Sigma_{s} l d s\right.\right. \\
& \left.\left.+\frac{\gamma}{\gamma-1} \operatorname{Tr}\left[\int_{0}^{T} \Lambda^{\top} \sqrt{\Sigma_{s}} d W_{s}\right]-\frac{\gamma}{\gamma-1} \int_{0}^{T} l^{\top} \sqrt{\Sigma_{s}} d B_{s}\right)\right] \\
= & \mathbb{E}^{\gamma}\left[\exp \left(-\frac{\gamma}{\gamma-1} r T+\frac{\gamma}{2(\gamma-1)^{2}} \operatorname{Tr}\left[\int_{0}^{T} \Lambda^{\top} \Sigma_{s} \Lambda d s\right]+\frac{\gamma}{2(\gamma-1)^{2}} \int_{0}^{T} l^{\top} \Sigma_{s} l d s\right)\right]
\end{aligned}
$$

where in the last equality we made the same change of measure $\mathbb{P} \rightarrow \mathbb{P}^{\gamma}$ as in the incomplete market case. Following the same reasoning we find that:

$$
J\left(t, X_{t}, \Sigma_{t}\right)=\frac{X_{t}^{\gamma}}{\gamma} e^{(1-\gamma) \operatorname{Tr}\left[A(t, T) \Sigma_{t}\right]+(1-\gamma) B(t, T)}
$$

where $A(t, T)$ and $B(t, T)$ are given by

$$
\begin{aligned}
& A(t, T)=P_{22}^{-1} P_{21} \\
& B(t, T)=-\frac{\beta}{2} \operatorname{Tr}\left[\log P_{22}+(T-t) \Gamma\right]-\frac{\gamma(T-t) r}{\gamma-1},
\end{aligned}
$$

with

$$
\left(\begin{array}{ll}
P_{11} & P_{12} \\
P_{21} & P_{22}
\end{array}\right)=\exp \left((T-t)\left(\begin{array}{cc}
\Gamma & -\Delta \\
K & -\Gamma^{\top}
\end{array}\right)\right)
$$




$$
\left\{\begin{array}{l}
\Gamma=M+\frac{\gamma}{\gamma-1} Q^{\top} \Lambda^{\top}, \\
\Delta=2 Q^{\top} Q \\
K=\frac{\gamma}{2(\gamma-1)^{2}}\left(\Lambda \Lambda^{\top}+l l^{\top}\right) .
\end{array}\right.
$$

\subsection{Proof of Proposition 3.5}

In order to determine the optimal weights $\left(\pi^{*}, \Pi^{*}\right)$, as in the incomplete market case from $X_{t}=H_{t}^{\frac{1}{\gamma-1}} e^{\operatorname{Tr}\left[A(t, T) \Sigma_{t}\right]+B(t, T)}$ we differentiate both sides and we equate the diffusions terms, thus obtaining:

$\operatorname{Tr}\left[\left(d Z_{t} \pi_{t}^{\top}+\frac{2}{\tau} d W_{t} Q \Phi_{t}\right) \sqrt{\Sigma}\right]=\operatorname{Tr}\left[\left(\frac{1}{\gamma-1} \Lambda+2 A Q^{\top}: \frac{-1}{\gamma-1} l\right) I_{n+1}(d W \vdots d B)^{\top} \sqrt{\Sigma}\right]$

where

$$
\Phi_{t}=\int_{0}^{\tau} e^{s M^{\mathbb{Q}}} \Pi_{t} e^{s M^{\mathbb{Q}}} d s
$$

Since the equality holds for all $\Sigma_{t}$ we deduce:

$$
\left(\pi_{t} \tilde{\rho}^{\top}+\frac{2}{\tau}\left(\Phi_{t} Q^{\top}: 0\right)\right)(d W: d B)^{\top}=\left(\frac{1}{\gamma-1} \Lambda+2 A Q^{\top}: \frac{-1}{\gamma-1} l\right)\left(\tilde{\rho} \tilde{\rho}^{\top}+\sum_{i=1}^{n} u_{i} u_{i}^{\top}\right)(d W: d B)^{\top},
$$

which leads to:

$\pi_{t} \tilde{\rho}^{\top}+\frac{2}{\tau}\left(\Phi_{t} Q^{\top} \vdots 0\right)\left(\tilde{\rho} \tilde{\rho}^{\top}+\sum_{i=1}^{n} u_{i} u_{i}^{\top}\right)=\left(\frac{1}{\gamma-1} \Lambda+2 A Q^{\top}: \frac{-1}{\gamma-1} l\right)\left(\tilde{\rho} \tilde{\rho}^{\top}+\sum_{i=1}^{n} u_{i} u_{i}^{\top}\right)$

Now by identifying the diffusion terms along the direction $\tilde{\rho}$ we get

$$
\pi_{t}+\frac{2}{\tau} \Phi_{t} Q^{\top} \rho=\frac{1}{\gamma-1} \Lambda \rho+2 A Q^{\top} \rho-\frac{\sqrt{1-\rho^{\top} \rho}}{\gamma-1} l=\frac{\lambda_{0}}{1-\gamma}+2 A Q^{\top} \rho
$$

while by projecting along the (orthogonal) direction $u_{n}$ we have

$$
\frac{2}{\tau} \Phi_{t} Q^{\top} \rho \frac{\sqrt{1-\rho^{\top} \rho}}{\sqrt{\rho^{\top} \rho}}=\left(\frac{1}{\gamma-1} \Lambda \rho+2 A Q^{\top} \rho\right) \frac{\sqrt{1-\rho^{\top} \rho}}{\sqrt{\rho^{\top} \rho}}+\frac{\sqrt{\rho^{\top} \rho}}{\gamma-1} l,
$$

which leads to

$$
\frac{2}{\tau} \Phi_{t} Q^{\top} \rho=2 A Q^{\top} \rho+\frac{1}{\gamma-1} \frac{1}{1-\rho^{\top} \rho}\left(\Lambda \rho+\lambda_{0} \rho^{\top} \rho\right) .
$$

Now we plug the previous expression into (49) and rearranging terms we arrive to

$$
\pi_{t}=-\frac{1}{\gamma-1} \frac{1}{1-\rho^{\top} \rho}\left(\lambda_{0}+\Lambda \rho\right) .
$$

In order to determine $\Phi$ we project the relation (49) along the (orthogonal) directions $u_{i} i=1, . ., n-1$, thus obtaining: 


$$
\frac{2}{\tau} \Phi_{t} Q^{\top} \alpha_{i} \alpha_{i}^{\top}=\frac{1}{\gamma-1} \Lambda \alpha_{i} \alpha_{i}^{\top}+2 A Q^{\top} \alpha_{i} \alpha_{i}^{\top}
$$

Now summing up the equalitites with respect of all directions and using the identity decomposition we arrive to the symmetric matrix representation for $\Phi$ :

$$
\frac{1}{\tau} \Phi_{t}=\frac{1}{4(\gamma-1)}\left(\Lambda\left(Q^{-1}\right)^{\top}+\left(Q^{-1}\right) \Lambda^{\top}+\frac{l \rho^{\top}}{\sqrt{1-\rho^{\top} \rho}}\left(Q^{-1}\right)^{\top}+Q^{-1} \frac{\rho l^{\top}}{\sqrt{1-\rho^{\top} \rho}}\right)+A,
$$

which gives the result. 


\begin{tabular}{cc}
\hline \hline & SP500/DAX \\
\hline \hline$M_{11}$ & -3.44 \\
Std. Dev. & $(0.239)$ \\
$M_{12}$ & 1.17 \\
Std. Dev. & $(0.211)$ \\
$M_{21}$ & 0.22 \\
Std. Dev. & $(0.469)$ \\
$M_{22}$ & -2.87 \\
Std. Dev. & $(0.21)$ \\
$Q_{11}$ & -0.05 \\
Std. Dev. & $(0.01)$ \\
$Q_{12}$ & -0.1 \\
Std. Dev. & $(0.012)$ \\
$Q_{21}$ & 0.07 \\
Std. Dev. & $(0.007)$ \\
$Q_{22}$ & 0.07 \\
Std. Dev. & $(0.014)$ \\
$\beta$ & 10.65 \\
Std. Dev. & $(0.733)$ \\
$\rho_{1}$ & 0.3 \\
Std. Dev. & $(0.067)$ \\
$\rho_{2}$ & -0.2 \\
Std. Dev. & $(0.071)$ \\
\hline \hline
\end{tabular}

Table 1: Estimation results of the WASC under the $\mathbb{P}$ probability measure.

\begin{tabular}{rc}
\hline \hline Parameter & Estimates \\
\hline \hline$\Lambda_{11}$ & -31.57 \\
Std.Dev. & $(-)$ \\
$\Lambda_{12}$ & 18.95 \\
Std.Dev. & $(-)$ \\
$\Lambda_{21}$ & 14.74 \\
Std.Dev. & $(-)$ \\
$\Lambda_{22}$ & -18.95 \\
Std.Dev. & $(-)$ \\
\hline \hline
\end{tabular}

Table 2: Variance risk premium matrix 
SP500 Descriptive statistics

\begin{tabular}{cccccc}
\hline \hline Maturity & Mean & Std. Dev. & Skewness & Kurtosis & Autocorrelation \\
\hline \hline 2 & 0.13 & 0.02 & 0.49 & 2.76 & 0.9988 \\
3 & 0.14 & 0.02 & 0.39 & 2.69 & 0.9991 \\
6 & 0.14 & 0.01 & 0.4 & 2.88 & 0.9996 \\
12 & 0.15 & 0.01 & 0.4 & 2.89 & 1.0001 \\
20 & 0.15 & 0.01 & 0.34 & 2.67 & 1.0002 \\
\hline \hline \multicolumn{5}{c}{ DAX Descriptive statistics } \\
\hline \hline Maturity & Mean & Std. Dev. & Skewness & Kurtosis & Autocorrelation \\
\hline \hline 2 & 0.18 & 0.02 & 0.25 & 2.67 & 0.9986 \\
3 & 0.18 & 0.02 & 0.26 & 2.76 & 0.999 \\
6 & 0.18 & 0.01 & 0.55 & 2.97 & 0.9995 \\
12 & 0.19 & 0.01 & 0.49 & 2.7 & 0.9997 \\
20 & 0.19 & 0.01 & 0.45 & 2.72 & 0.9997 \\
\hline \hline
\end{tabular}

Table 3: Descriptive statistics for variance swap prices

\begin{tabular}{cccccc}
\hline \hline \multicolumn{7}{c}{ SP500 Descriptive statistics } \\
\hline \hline Maturity & Mean & RMSE & Autocorrelation & Max & R2 \\
2 & -0.14 & 1.01 & 0.59 & 5.95 & 0.68 \\
3 & 0.11 & 0.84 & 0.57 & 5.22 & 0.71 \\
6 & 0.15 & 0.56 & 0.51 & 3.55 & 0.76 \\
12 & -0.05 & 0.41 & 0.56 & 2.47 & 0.75 \\
20 & -0.03 & 0.4 & 0.75 & 1.84 & 0.65 \\
Average & -0.02 & 0.61 & 0.596 & 5.95 & 0.71 \\
\hline \hline \multicolumn{7}{c}{ DAX Descriptive statistics } & & \\
\hline \hline Maturity & Mean & RMSE & Autocorrelation & Max & $\mathbf{R 2}$ \\
2 & 0.1 & 0.8 & 0.28 & 3.38 & 0.86 \\
3 & 0.14 & 0.69 & 0.31 & 2.69 & 0.86 \\
6 & 0.09 & 0.51 & 0.42 & 1.91 & 0.86 \\
12 & -0.14 & 0.51 & 0.76 & 1.54 & 0.87 \\
20 & -0.1 & 0.62 & 0.9 & 1.63 & 0.86 \\
Average & -0.04 & 0.63 & 0.534 & 3.38 & 0.81 \\
\hline \hline
\end{tabular}

Table 4: Descriptive statistics for variance swap pricing errors 


\begin{tabular}{c|cc|cc|cc}
\hline \hline & \multicolumn{2}{|c|}{ Declared BPT results } & \multicolumn{2}{|c|}{ Correct BPT results } & \multicolumn{2}{c|}{ WASC results } \\
\hline \hline & SP500 & DAX & SP500 & DAX & SP500 & DAX \\
\hline \hline Total & 10 & 11 & 0.77 & 1.01 & 0.63 & 0.89 \\
Vol Hedging & 1.5 & 2 & 0.35 & 0.55 & 0.27 & 0.53 \\
Covol hedging & 2.5 & 3 & 0.06 & 0.44 & 0.15 & 0.18 \\
Correlation Hedging & 6 & 6 & 0.36 & 0.01 & 0.21 & 0.19 \\
\hline \hline
\end{tabular}

Table 5: Detailed decomposition of intertemporal hedging demand on both assets in terms of percent over the myopic startegy

To recompute the Buraschi et al. (2006) solution, we used the estimates provided in the version of their paper issued on March 19th, 2007. The values for $M, Q$ and $\beta$ are:

$$
M=\left(\begin{array}{cc}
-0.2816 & 0.0147 \\
0.0502 & -0.1548
\end{array}\right) Q=\left(\begin{array}{cc}
-0.0009 & -0.0030 \\
0.0028 & 0.0022
\end{array}\right) \beta=10
$$

The sample moments presented in Buraschi et al. (2006) for the returns are:

$$
\mu=\left(\begin{array}{c}
0.135 \\
0.1106
\end{array}\right) \Sigma=\left(\begin{array}{c}
0.1597 \\
0.2013
\end{array}\right) \rho^{\mathrm{SP} / \mathrm{DAX}}=0.3672
$$

The risk-free rate has been chosen as the average risk-free rate over their estimation period. The solution that we recomputed proved not to be very sensitive to the model's parameters, but the $Q$ matrix. This is not a surprise since $Q$ is controlling the volatility risk: should this matrix be multiplied by 10 - without changing the remaining parameters - and the results obtained would be close to what is obtained in the Buraschi et al. (2006) paper. However, in order not to change the distributional properties of the estimated process, $M$ should also be scaled accordingly. In such a case, the hedging ratio is again far below $5 \%$.

\begin{tabular}{ccc}
\hline \hline & SP500 & DAX \\
\hline \hline$\mu$ & 0.0793 & 0.0821 \\
$r_{f}$ & 0.0496 & 0.0411 \\
\hline \hline
\end{tabular}

Table 6: Risk free rate and estimated drift

\begin{tabular}{cc}
\hline \hline Parameter & Estimates \\
\hline \hline$\Sigma_{11}$ & 0.02359 \\
$\Sigma_{21}$ & 0.01505 \\
$\Sigma_{22}$ & 0.04762 \\
\hline \hline
\end{tabular}

Table 7: Initial Value for $\Sigma_{0}$

\begin{tabular}{rc}
\hline \hline & Hedging ratios \\
\hline \hline SP500 & -4.7395 \\
DAX & 3.3667 \\
\hline
\end{tabular}

Table 8: Heding demand in the complete market case 


\begin{tabular}{ccc}
\hline \hline \multicolumn{3}{c}{ Hedging Ratios } \\
& Incomplete & Complete \\
\hline \hline$\pi^{1}$ & 0.2995 & -4.7395 \\
$\pi^{2}$ & 0.1939 & 3.3667 \\
$\Pi^{11}$ & - & -58.301 \\
$\Pi^{12}$ & - & -77.135 \\
$\Pi^{22}$ & - & 53.245 \\
\hline \hline
\end{tabular}

Table 9: Hedging ratios for the assets within the two models.

$\pi^{1}$ (resp. $\pi^{2}$ ) is the hedging ratio for the first (resp. second) asset. $\Pi^{11}$ (resp. $\Pi^{22}$ ) stands for the hedging ratio for the variance swap on the first (resp. second) asset and $\Pi^{12}$ is the hedging ratio for the covariance swap contract. 


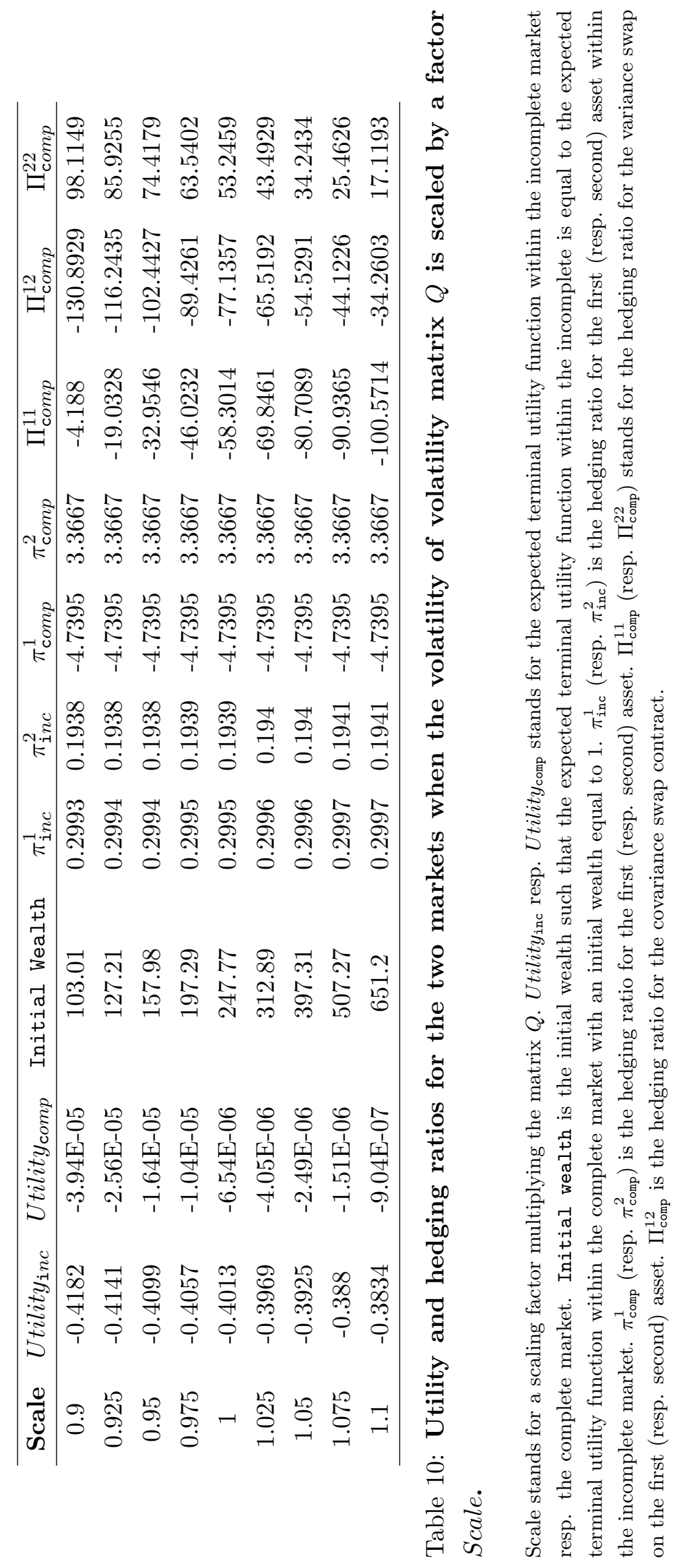




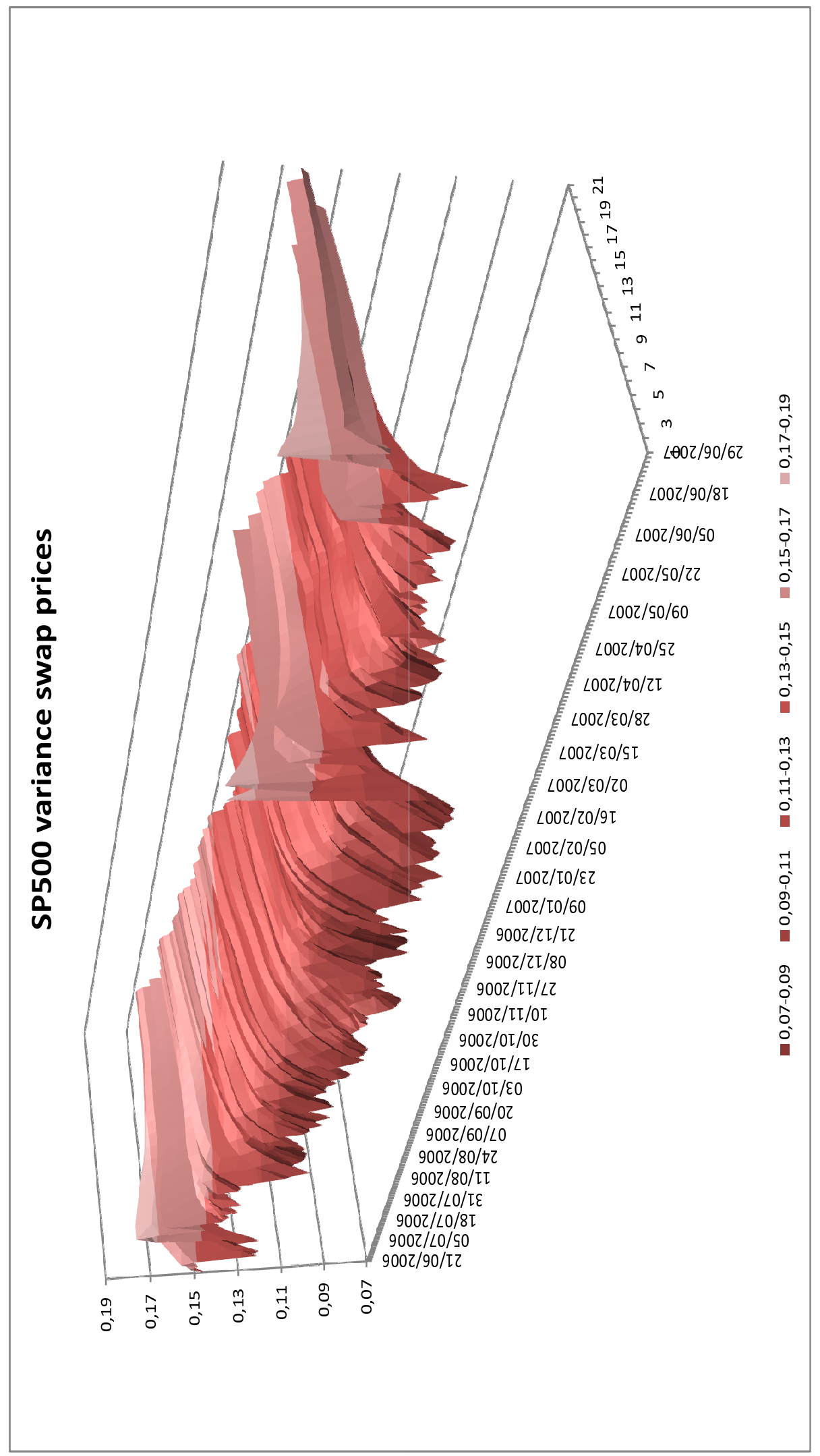

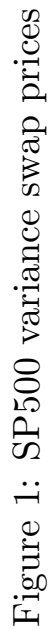




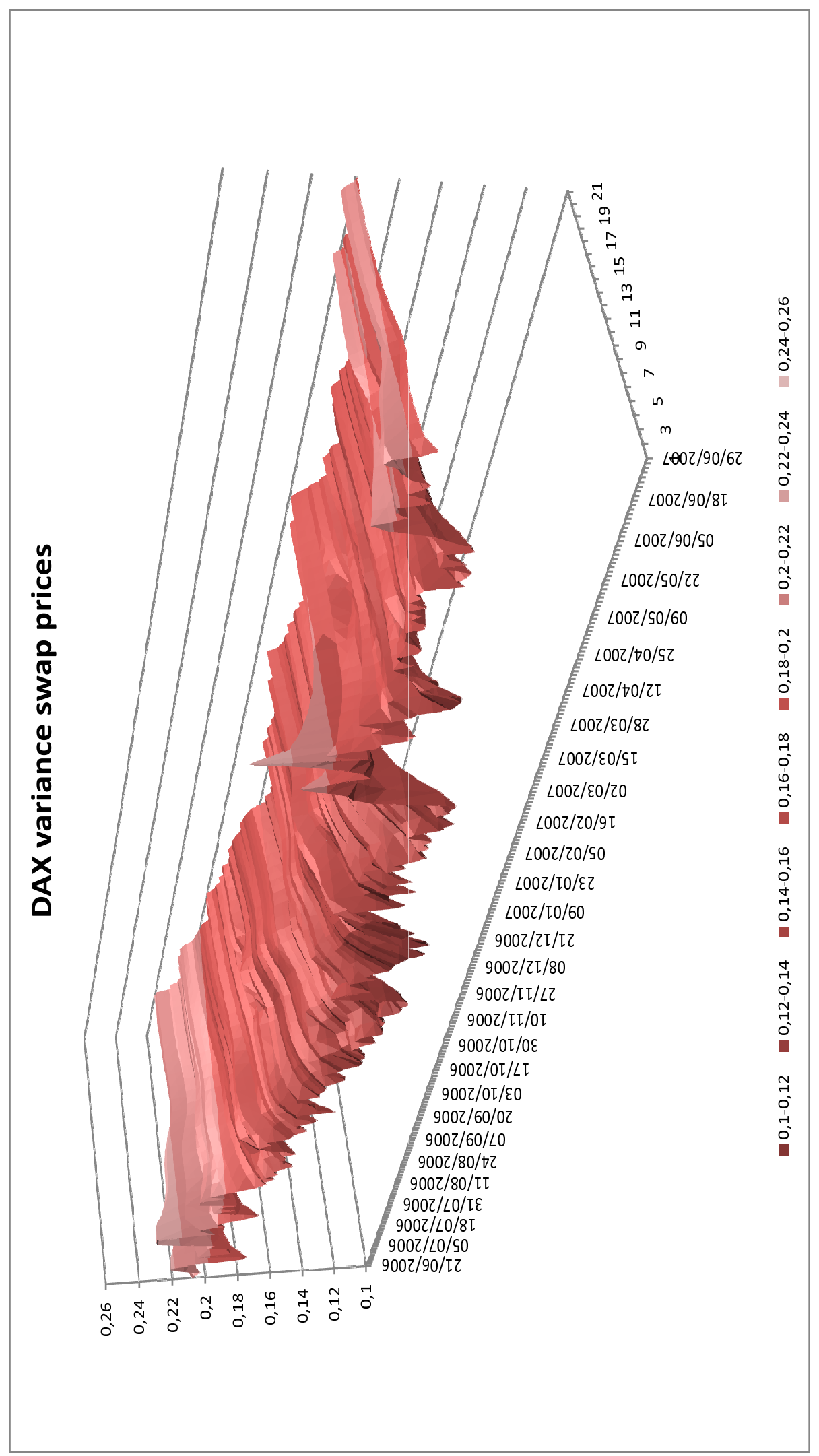

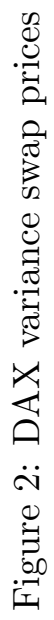




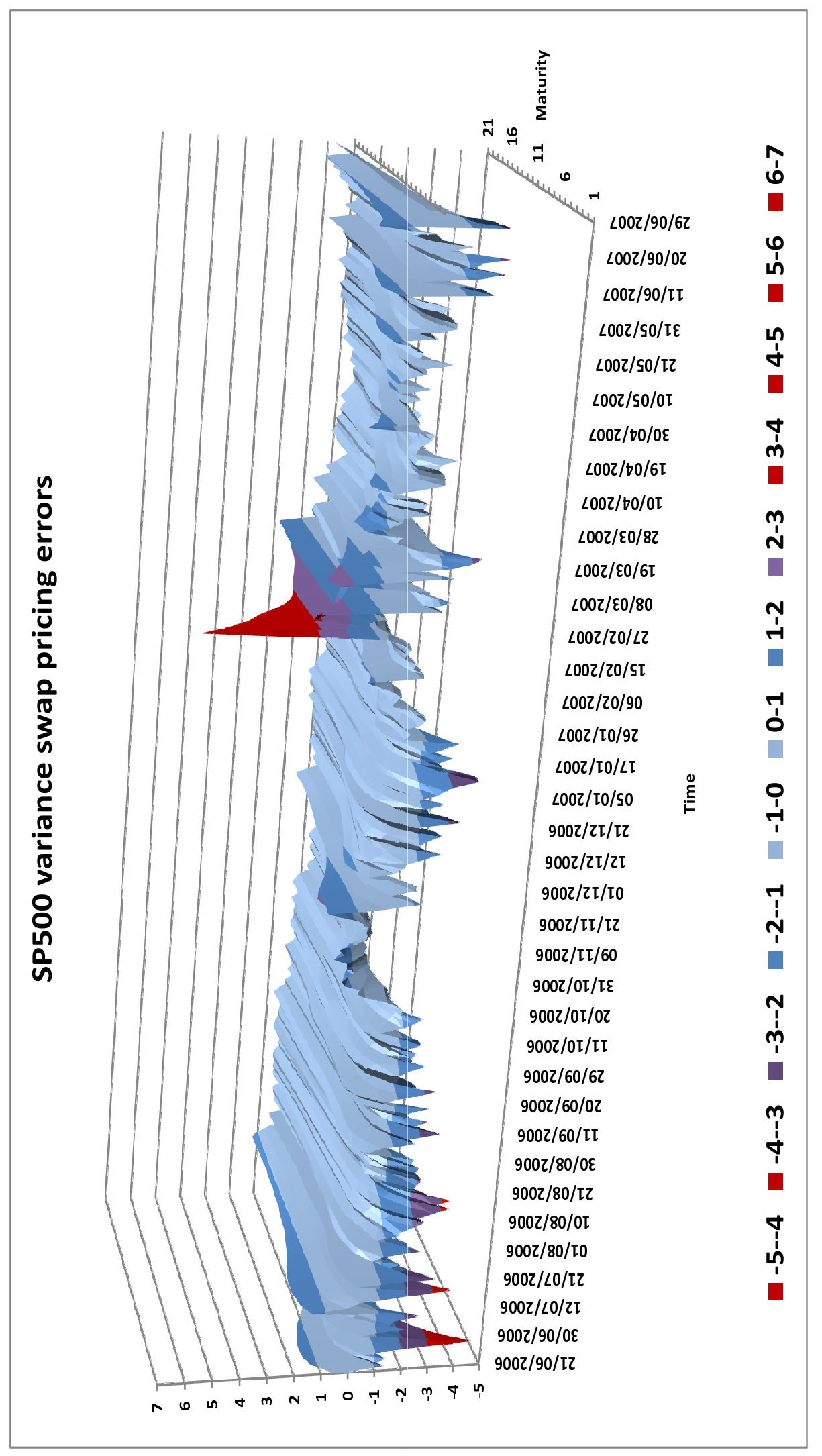




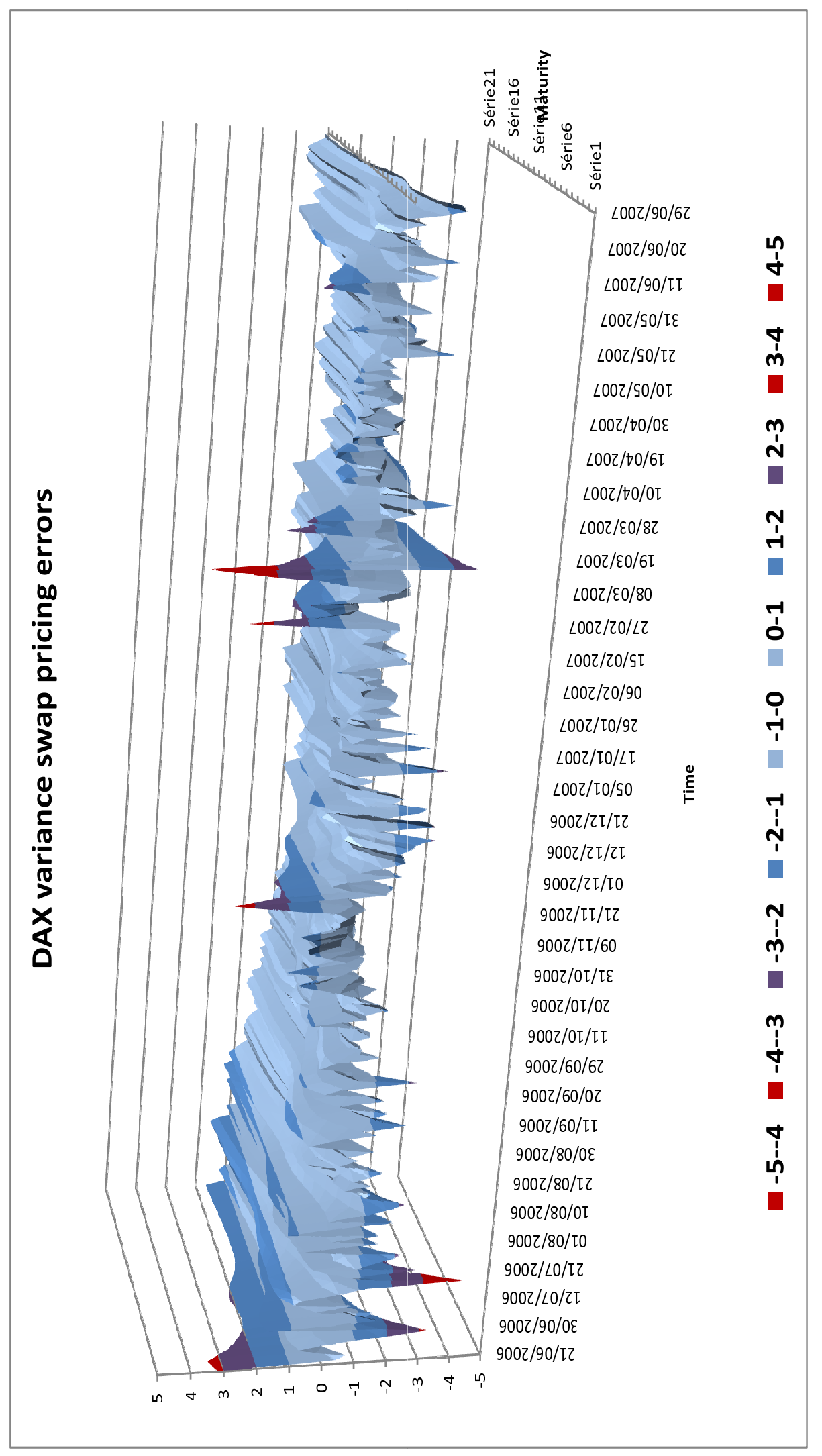

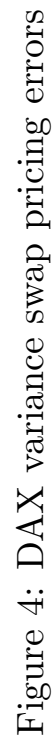




\section{References}

Brandt, M. W. and He, P. (2006). Simulated Likelihood Estimation of Affine Term Structure Models from Panel Data. SSRN-885682.

Bru, M. F. (1991). Wishart Processes. Journal of Theoretical Probability, 4:725-743.

Buraschi, A., Porchia, P., and Trojani, F. (2006). Correlation risk and optimal portfolio choice. Working paper, SSRN-908664.

Carr, P. and Corso, A. (2001). Commodity Covariance Contracting. Energy Risk-April, pages $42-45$.

Carrasco, M., Chernov, M., Florens, J.-P., and Ghysels, E. (2007). Efficient Estimation of Jump Diffusions and General Dynamic Models with a Continuum of Moment Conditions. Journal of Econometrics, (140):529-573.

Chacko, G. and Viceira, L. M. (2005). Dynamic Consumption and Portfolio Choice with Stochastic Volatility in Incomplete Markets. Review of Financial Studies, 18(4):13691402 .

Da Fonseca, J., Grasselli, M., and Ielpo, F. (2007a). Estimating the Wishart Affine Stochastic Correlation Model Using the Empirical Characteristic Function. SSRN1054721.

Da Fonseca, J., Grasselli, M., and Tebaldi, C. (2007b). Option pricing when correlations are stochastic: an analytical framework. Review of Derivatives Research, 10(2):151180.

Davis, M. and Obloj, J. (2007). Market completion using options. Working paper, arXiv:0710.2792.

Duffee, G. and Stanton, R. (2004). Estimation of Dynamic Term Structure Models. Working paper.

Duffee, G. R. (2002). Term premia and interest rate forecasts in affine models. Journal of Finance, 57:405-443.

Gourieroux, C. and Sufana, R. (2004). Derivative Pricing with Multivariate Stochastic Volatility: Application to Credit Risk. Working paper.

Grasselli, M. and Tebaldi, C. (2008). Solvable Affine Term Structure Models. Mathematical Finance, 18:135-153.

He, H. and Pearson, N. (1991). Consumption and Portfolio Policies with Incomplete Markets and Short-Sale Constraints: The Infinite Dimensional Case. Journal of Economic Theory, 54:239-250. 
Heston, S. (1993). A Closed-Form Solution for Options with Stochastic Volatility with Applications to Bond and Currency Options. The Review of Financial Studies, 6(2):327-343.

Hobson, D. G. (2004). Stochastic Volatility Models, Correlation, and the q-Optimal Measure. Mathematical Finance, 14(4):537-556.

Hull, J. C. (2008). Options, Futures, and Other Derivatives (7th Edition). Prentice Hall.

Karatzas, I. and Shreve, S. E. (1998). Methods of Mathematical Finance. SpringerVerlag.

Leippold, M., Egloff, D., and Wu, L. (2007). Variance Risk Dynamics, Variance Risk Premia, and Optimal Variance Swap Investments. SSRN-903728.

Liu, J. (2005). Portfolio Selection in Stochastic Environments. Working paper UCLA.

Liu, J. (2007). Portfolio Selection in Stochastic Environments. Review of Financial Studies, 20(1):1-39.

Merton, R. C. (1971). Optimum consumption and portfolio rules in a continuous-time model. Journal of Economic Theory, 3(4):373-413. 